COMMUNICATIONS IN

NUMBER THEORY AND PHYSICS

Volume 5, Number 1, 57-100, 2011

\title{
Archimedean $L$-factors and topological field theories I
}

\author{
Anton Gerasimov, Dimitri Lebedev and Sergey Oblezin
}

\begin{abstract}
We propose a functional integral representation for Archimedean $L$-factors given by products of $\Gamma$-functions. The corresponding functional integral arises in the description of type-A equivariant topological linear sigma model on a disk. The functional integral representation provides in particular an interpretation of the $\Gamma$-function as an equivariant symplectic volume of an infinitedimensional space of holomorphic maps of the disk to $\mathbb{C}$. This should be considered as a mirror dual to the classical Euler integral representation of the $\Gamma$-function. We give an analogous functional integral representation of $q$-deformed $\Gamma$-functions using a three-dimensional equivariant topological linear sigma model on a handlebody. In general, upon proper ultra violent completion, the topological sigma model considered on a particular class of threedimensional spaces with a compact Kähler target space provides a quantum field theory description of a $K$-theory version of GromovWitten invariants.
\end{abstract}

\section{Introduction}

Archimedean local $L$-factors were introduced to simplify functional equations of global $L$-functions. From the point of view of arithmetic geometry these factors complete the Euler product representation of global $L$-factors by taking into account Archimedean places of the compactified spectrum of global fields. A known construction of non-Archimedean local $L$-factors is rather transparent and uses characteristic polynomials of the image of the Frobenius homomorphism in finite-dimensional representations of the local Weil-Deligne group closely related to the local Galois group. On the other hand, Archimedean $L$-factors are expressed through products of $\Gamma$-functions and thus are analytic objects avoiding simple algebraic interpretation. Moreover, Archimedean Weil-Deligne groups are rather mysterious objects in comparison with their non-Archimedean counterparts. For instance, in the case of the field of complex numbers the corresponding Galois group is trivial while the Weil-Deligne group is isomorphic to the multiplicative group $\mathbb{C}^{*}$ of 
complex numbers. In many cases the multiplicative group $\mathbb{C}^{*}$ effectively plays the role of the Galois group for complex numbers $[6,12]$. Thus, for instance, by analogy with the action of the Galois group Gal $\left(\overline{\mathbb{F}}_{p} / \mathbb{F}_{p}\right)$ on étale cohomology of schemes over $\overline{\mathbb{F}}_{p}$, the multiplicative group $\mathbb{C}^{*}$ acts on the complexified cohomology of compact non-singular complex algebraic varieties providing the standard Hodge decomposition. The Archimedean Weil-Deligne groups also play an important role in a formulation of Archimedean local Langlands correspondence (see e.g. [1]).

In a series of papers [21-23], we approach the problem of a proper interpretation of Archimedean $L$-factors and in particular of Archimedean Weil-Deligne group using our previous results on relations between quantum integrable systems and representation theory as well as results of [27-29,31] on quantum cohomology and quantum $K$-theory. In [21], we propose an explicit description of the Archimedean local Hecke algebras in terms of intertwining integral operators. These integral operators are instances of the Baxter operator playing an important role in the construction of explicit solutions of quantum integrable systems. We demonstrate that, by analogy with the generators of non-Archimedean Hecke algebras, the common eigenfunctions of the Baxter operators are given by "class one" Whittaker functions. Remarkably the corresponding eigenvalues are precisely Archimedean $L$-factors in complete analogy with a non-Archimedean case. Here the "class one" condition means that the Whittaker function rapidly decreases outside some domain and is an Archimedean counterpart of the non-Archimedean "class one" Whittaker function from [10,40].

In [30] Givental introduced new integral representations of the Whittaker functions. These representation arise naturally in the description of the generating functions of the equivariant Gromov-Witten (GW) invariants of the flag spaces. In [31] a $K$-theory analog of the $\mathrm{GW}$-invariants was considered and it was shown that the generating function of $K$-theory GW-invariants are given by $q$-deformed Whittaker functions. However, one should note that the Whittaker functions and their $q$-deformations considered in the framework of GW theory [35] are not "class one" Whittaker functions and thus do not have direct arithmetic interpretation. In [25], we show that Baxter integral operators appear as an essential ingredient of the Givental integral representations of the Whittaker functions.

The relevance of the Baxter integral operators both to the description of Archimedean Hecke algebra and GW invariants implies a relation between these two subjects. In [23], we confirm these expectations. We construct explicit expressions of $q$-deformed "class one" Whittaker functions, $q$-analogs of Baxter operators, $q$-analogs of Archimedean $L$-factors and relate these constructions to counting problems on the moduli space of holomorphic 
maps of $\mathbb{P}^{1}$ to the flag manifolds. In particular $q$-deformed $L$-factors and $q$-deformed "class one" Whittaker functions corresponding to $(\ell+1)$ dimensional representations of the Archimedean Weil-Deligne group $\mathbb{C}^{*}$ are given by characters of $\mathbb{C}^{*} \times G L_{\ell+1}(\mathbb{C})$ modules realized as cohomology of holomorphic line bundles on the moduli space of holomorphic maps of $\mathbb{P}^{1}$. Thus for $q$-deformed $L$-factor, the corresponding $\mathbb{C}^{*} \times G L_{\ell+1}(\mathbb{C})$-module is given by a limit of the space of degree $d$ holomorphic polynomial maps of $\mathbb{C}$ to $\mathbb{C}^{\ell+1}$ when $d \rightarrow \infty$. Here $\mathbb{C}^{*}$ acts on $\mathbb{C}$ by multiplication and $G L_{\ell+1}(\mathbb{C})$ acts on $\mathbb{C}^{\ell+1}$ through the standard representation. We stress in [23] that the constructed $q$-deformed local $L$-factors/Whittaker functions interpolate Archimedean and non-Archimedean local $L$-factors/Whittaker functions. Non-Archimedean $L$-factor associated to an $(\ell+1)$-dimensional representation $V=\mathbb{C}^{\ell+1}$ of the Weil-Deligne group can be identified with a character of a direct sum of symmetric powers of $V$. Similarly, according to $[10,40]$, $p$-adic "class one" Whittaker functions for a reductive algebraic group $G$ are given by characters of irreducible finite-dimensional representations of the Langlands dual group ${ }^{L} G_{0}$ (Shintani-Casselman-Shalika (SCS) formula). SCS formula essentially uses homomorphims of the local Weil-Deligne group (simple modification of the local Galois group $\operatorname{Gal}\left(\overline{\mathbb{Q}}_{p} / \mathbb{Q}_{p}\right)$ ) to ${ }^{L} G_{0}$ and thus provides an arithmetic construction of $p$-adic Whittaker function. Remarkably, the representation [23] of the $q$-deformed Whittaker function as a character of a $\mathbb{C}^{*} \times G L_{\ell+1}(\mathbb{C})$-module reduces to SCS-formula in the appropriate limit. Under the same limit $q$-deformed $L$-factor reduces to non-Archimedean $L$-factors. Although the interpolation and limiting procedure allow us to relate constructions of local Archimedean and non-Archimedean $L$-factors a direct analytic construction of the Archimedean $L$-factors given by the product of $\Gamma$-functions was missing.

In this paper, we provide an explicit construction of Archimedean $L$-factors as functional integrals in equivariant type A topological linear sigma model on a disk. We remark in [23] that the construction of the "class one" q-deformed Whittaker function implies consideration of holomorphic maps of a disk to flag spaces in contrast with [30,31] where holomorphic maps of $\mathbb{P}^{1}$ into flag spaces were considered. The type A topological sigma model considered below is precisely of the kind that describes GW-invariants. Specifically, we consider $S^{1} \times U_{\ell+1}$-equivariant type A topological linear sigma model on $D=\{z|| z \mid \leq 1\}$ with the target space $V=\mathbb{C}^{\ell+1}$ deformed by a boundary observable. The target space here is a finite-dimensional space $V$ appearing in the standard construction of an Archimedean $L$-factor in terms of a finite-dimensional representation of the Archimedean Weil-Deligne group $\mathbb{C}^{*}$. The group $S^{1}$ acts by rotations on $D$ and $U_{\ell+1}$ acts in $\mathbb{C}^{\ell+1}$ via the standard representation. The functional 
integral representation for local $L$-factors is given in Theorem 2.1. Let us stress that the underlying action of the considered topological theory is quadratic and the functional integral allows mathematical formulation using $\zeta$-function regularization $[32,38]$.

The functional integral representation of local Archimedean $L$-factors proposed in this note can be interpreted as a calculation of an equivariant symplectic volume of the space of holomorphic maps of a disk $D$ into complex vector spaces. In particular, classical $\Gamma$-function coincides with an equivariant volume of the space of holomorphic maps of $D$ to $\mathbb{C}$. It appears that the infinite-dimensional symplectic geometry is a proper framework for a mysterious geometry over Archimedean places.

The interpretation in terms of equivariant symplectic volumes provides a natural way to deform $\Gamma$-function. According to standard arguments in quantum mechanics equivariant symplectic volume can be interpreted as a classical limit of the partition function of the quantum system obtained by a quantization of the symplectic manifold. We apply this reasoning to the space of holomorphic maps of $D$ to $\mathbb{C}$ and obtain a canonical quantization of $\Gamma$-functions and therefore of local Archimedean $L$-factors. Fortunately, thus constructed quantum analogs of $\Gamma$-function and local Archimedean $L$-factors coincide with $q$-deformations of their classical counterparts [22,23]. For these $q$-deformed/quantum local Archimedean $L$-factors we provide a representation in terms of the functional integral in three-dimensional equivariant topological linear sigma model on a handlebody $S^{1} \times D$. We also argue that upon a proper ultraviolent completion the constructed three-dimensional topological sigma model on a special type of compact three-dimensional spaces with a Kähler target space provides a description of quantum $K$ theory invariants [29,31].

Let us finally refer to the various previous discussions of hidden structures behind local Archimedean $L$-factors related with our proposal. The point of view that is most close to the approach of this note was advocated by Deninger [13,14] (see also [37]). Namely, Deninger proposed an interpretation of local Archimedean $L$-factors as regularized infinite-dimensional determinants. Corresponding infinite-dimensional vector spaces can be identified with cohomology groups of algebraic manifold with coefficients in an Archimedean analog of the rings introduced by Fontaine [18] for a description of the $p$-adic Hodge periods. Let us note also that equivariant symplectic volumes of the space of maps of a disk into symplectic manifolds were discussed in [27] in connection with GW theory. Also Vojta [43] proposed a detailed set of analogies between number theory and value distribution theory of holomorphic functions due to Nevanlinna. Finally, long ago 
Deligne considered an analogy between schemes over $\mathbb{Q}_{p}$ allowing good integer models and families of complex manifolds over a disk [12]. This analogy motivated the development of the theory of mixed Hodge structures.

The plan of the paper is as follows. In Section 1, we recall two basic constructions of local $L$-factors. In Section 2, we prove the main statement of the paper (Theorem 2.1) by identifying Archimedean $L$-factors with a particular functional integral in the equivariant type A topological linear sigma model on a disk. In Section 3, we provide interpretation of the functional integral as an equivariant symplectic volume of the space of holomorphic maps of the disk into complex vector space. In Section 4, we consider a three-dimensional topological sigma model calculating quantum $K$-theory invariants introduced in $[29,31]$. We demonstrate that the functional integral in the three-dimensional equivariant topological linear sigma model on the handle-body reproduces $q$-version of Archimedean $L$-factor introduced in $[22,23]$. In Section 5, we conclude with some general remarks and discuss further directions of research. In the appendix, the standard facts about $\zeta$-regularization of infinite-dimensional Gaussian integrals are collected.

\section{Two constructions of $L$-factors}

To put our results in the right perspective we briefly recall in this section some standard facts on local and global $L$-functions (see e.g. $[1,41,42]$ ). This section does not contain new results and can be skipped by a connoisseur. For standard definitions and elementary detailed discussion, see for example $[9,36]$.

Two essentially different constructions of $L$-functions are known. The first one uses automorphic representations of reductive algebraic groups. In this construction, one expresses $L$-functions in terms of a spectrum of the corresponding Hecke algebra. In the case of a local field $F$, we consider representations of the group $G(F)$ and in the case of a global field $F$ the group $G$ is defined over adeles $\mathbb{A}_{F}$. The second construction of $L$-factors is based on arithmetic properties of the base field $F$ and uses homomorphims of its Galois group (more precisely Weil-Deligne group) into the Langlands dual group ${ }^{L} G$. Here the Langlands dual group ${ }^{L} G$ for a field $F$ is defined as an extension of the Galois group $\operatorname{Gal}(\bar{F} / F)$ by a dual algebraic group ${ }^{L} G_{0}(\bar{F})$. The Langlands correspondence claims that both automorphic and arithmetic constructions lead to the same set of $L$-functions.

Let us describe these two constructions of $L$-functions for the base field $F=\mathbb{Q}$ in more details. We start with the automorphic construction of $L$-functions. Let $\mathbb{A}$ be the adele ring of $\mathbb{Q}$ and $G$ be a reductive algebraic 
group. An automorphic representation $\pi$ of $G(\mathbb{A})$ is an irreducible representation entering the decomposition of the left representation of $G(\mathbb{A})$ in $L^{2}(G(\mathbb{A}) / G(\mathbb{K}))$, where $G(\mathbb{K})$ is a maximal compact subgroup in $G(\mathbb{A})$. The representation $\pi$ can be characterized by an automorphic form $\phi$, such that it is an eigenfunction of any element of the global Hecke algebra $\mathcal{H}(G(\mathbb{A}))$. The global Hecke algebra has natural structure of a product $\mathcal{H}(G(\mathbb{A}))=$ $\left(\otimes_{p} \mathcal{H}_{p}\right) \otimes \mathcal{H}_{\infty}$ of local non-Archimedean Hecke algebras $\mathcal{H}_{p}=\mathcal{H}\left(G\left(\mathbb{Q}_{p}\right)\right.$, $\left.G\left(\mathbb{Z}_{p}\right)\right)$ for each prime $p$ and an Archimedean Hecke algebra $\mathcal{H}_{\infty}=\mathcal{H}(G(\mathbb{R})$, $K)$, where $K$ is a maximal compact subgroup in $G(\mathbb{R})$. The local Hecke algebra $\mathcal{H}_{p}$ is isomorphic to the algebra of characters of finite-dimensional representations of a Langlands dual group ${ }^{L} G$, (e.g. for ${ }^{L} G_{0}$ being $A_{\ell}, B_{\ell}, C_{\ell}$, $D_{\ell}$ the duals are $A_{\ell}, C_{\ell}, B_{\ell}, D_{\ell}$ respectively). For each unramified representation of $G\left(\mathbb{Q}_{p}\right)$ one can define an action of $\mathcal{H}_{p}$ such that the automorphic form $\phi$ is a common eigenfunction of all elements of $\mathcal{H}_{p}$ for all primes $p$ and thus defines a set of homomorphisms $\mathcal{H}_{p} \rightarrow \mathbb{C}$. Identifying local Hecke algebras with the algebra of characters of finite-dimensional representations of ${ }^{L} G$ one can describe this set of homomorphisms as a set of conjugacy classes $g_{p}$ in ${ }^{L} G$. Given a complex finite-dimensional representation $\rho_{V}:{ }^{L} G \rightarrow G L(V, \mathbb{C})$ one can construct $L$-function corresponding to the automorphic form $\phi$ as the Euler product

$$
L\left(s, \phi, \rho_{V}\right)=\prod_{p}^{\prime} L_{p}\left(s, \phi, \rho_{V}\right)=\prod_{p}^{\prime} \operatorname{det}_{V}\left(1-\rho_{V}\left(g_{p}\right) p^{-s}\right)^{-1},
$$

where $\prod_{p}^{\prime}$ is a product over the primes $p$ such that the corresponding representation of $G\left(\mathbb{Q}_{p}\right)$ is not ramified. It is natural to complete the product by including local $L$-factors corresponding to Archimedean and ramified places. We omit a discussion of $L$-factors for ramified representations and consider the construction of Archimedean $L$-factors. For Archimedean places the Hecke eigenfunction property is usually replaced by the eigenfunction property with respect to a ring of invariant differential operators on $G(\mathbb{R})$. The corresponding eigenvalues are described by a conjugacy class $t_{\infty}$ in the Lie algebra ${ }^{L} \mathfrak{g}_{0}=\operatorname{Lie}\left({ }^{L} G_{0}\right)$. The Archimedean $L$-factor is then given by a product of $\Gamma$-functions

$$
\begin{aligned}
L_{\mathbb{R}}\left(s, \phi, \rho_{V}\right) & =\prod_{j=1}^{\ell+1} \pi^{-\frac{s-\alpha_{j}}{2}} \Gamma\left(\frac{s-\alpha_{j}}{2}\right) \\
& =\operatorname{det}_{V} \pi^{-\frac{s-\rho_{V}\left(t_{\infty}\right)}{2}} \Gamma\left(\frac{s-\rho_{V}\left(t_{\infty}\right)}{2}\right),
\end{aligned}
$$


where $\rho:{ }^{L} G_{0} \rightarrow G L_{\ell+1}(\mathbb{C})$ and $\rho_{V}\left(t_{\infty}\right)=\operatorname{diag}\left(\alpha_{1}, \ldots, \alpha_{\ell+1}\right)$. In [21], we demonstrate that the eigenvalue property with respect to the ring of invariant differential operators on $G(\mathbb{R})$ can be equivalently replaced by the eigenvalue property with respect to a set of integral operators. These operators generate Archimedean Hecke algebra $\mathcal{H}(G(\mathbb{R}), K)$, where $K$ is a maximal compact subgroup of $G(\mathbb{R})$. Elements $\mathcal{H}(G(\mathbb{R}), K)$ are $K$-biinvariant functions on $G(\mathbb{R})$ and the structure of the algebra is given by a convolution. This completely restores the symmetry between Archimedean and non-Archimedean places. The local Archimedean $L$-factors (1.2) correspond to the real place of $\mathbb{Q}$. For more general global fields complex Archimedean places appear and the corresponding complex local $L$-factors are given by analogous expressions

$$
L_{\mathbb{C}}\left(s, \phi, \rho_{V}\right)=\operatorname{det}_{V}(2 \pi)^{-\left(s-\rho_{V}\left(t_{\infty}\right)\right)} \Gamma\left(s-\rho_{V}\left(t_{\infty}\right)\right) .
$$

The global $L$-function can be defined as an analytic continuation of the product

$$
\Lambda(s, \phi, \rho)=L(s, \phi, \rho) L_{\infty}(s, \phi, \rho)
$$

where $L_{\infty}(s)$ is a product of local factors corresponding to Archimedean places. Global $L$-functions should satisfy a functional equation

$$
\Lambda(1-s, \phi, \rho)=\epsilon(s, \phi, \rho) \Lambda\left(s, \phi_{\pi^{\vee}}, \rho^{\vee}\right)
$$

where the $\epsilon$-factor is of the form $\epsilon(s, \phi, \rho)=A B^{s}, A \in \mathbb{C}^{*}, B \in \mathbb{R}_{+}$and $\pi^{\vee}$, $\rho^{\vee}$ are dual to $\pi, \rho$.

Now let us describe another construction of the local $L$-functions based on arithmetic properties of a base field $F$. To discuss both Archimedean and non-Archimedean cases in the same setting one should introduce a notion of the Weil-Deligne group generalizing the standard notion of the Galois group. Let $\bar{F}$ be an algebraic closure of a local number field $F$. The Weil group $W_{F}$ of $F$ should satisfy the following properties. First, there should exist a homomorphism with a dense image in the natural topology on $\operatorname{Gal}(\bar{F} / F)$

$$
\phi: W_{F} \rightarrow \operatorname{Gal}(\bar{F} / F)
$$

Second, for any Galois extension $E$ of $F$, there should be an inclusion in the abelianization $W_{E}^{a b}=W_{E} /\left[W_{E}, W_{E}\right]$ of $W_{E}$

$$
r: E^{*} \rightarrow W_{E}^{a b}
$$


such that the composition

$$
E^{*} \rightarrow \mathrm{Gal}^{a b}(\bar{F} / E)
$$

is a basic homomorphism of abelian class field theory.

In the case of the local field $F=\mathbb{Q}_{p}$ this boils down to the following construction. Let $\mathbb{F}_{p}$ be a residue field of $\mathbb{Q}_{p}$. Then we have the extension

$$
0 \longrightarrow I_{p} \longrightarrow \operatorname{Gal}\left(\overline{\mathbb{Q}}_{p} / \mathbb{Q}_{p}\right) \longrightarrow \operatorname{Gal}\left(\overline{\mathbb{F}}_{p} / \mathbb{F}_{p}\right) \longrightarrow 0
$$

where $I_{p}$ is the inertia group and $\operatorname{Gal}\left(\overline{\mathbb{F}}_{p} / \mathbb{F}_{p}\right)$ is a pro-finite completion of the cyclic groups with the generator given by Frobenius homomorphism $F r_{p}$ : $x \rightarrow x^{p}$. The Weyl group $W_{\mathbb{Q}_{p}}$ is then a subgroup of $\operatorname{Gal}\left(\overline{\mathbb{Q}}_{p} / \mathbb{Q}_{p}\right)$ consisting of the elements such that their image in $\operatorname{Gal}\left(\overline{\mathbb{F}}_{p} / \mathbb{F}_{p}\right)$ is an integer power of Frobenius $F r_{p}$. Maximal abelian subgroup of $W_{\mathbb{Q}_{p}}$ is canonically identified with $\mathbb{Q}_{p}^{*}$.

For the field $F=\mathbb{C}$ of complex numbers, the Galois group $\mathrm{Gal}(\overline{\mathbb{C}} / \mathbb{C})$ is trivial and $W_{\mathbb{C}}=\mathbb{C}^{*}$. The homomorphism $\phi$ is trivial and $r$ is the identity map. For real numbers $F=\mathbb{R}$, the Galois group $\operatorname{Gal}(\mathbb{C} / \mathbb{R})=\mathbb{Z}_{2}$ is generated by complex conjugation $F r_{\infty}$ and the Weil group $W_{\mathbb{R}}=\mathbb{C}^{*} \cup j \mathbb{C}^{*}$ is generated by a copy of $\mathbb{C}^{*}$ and an element $j$, subjected to the relations:

$$
j x j^{-1}=\bar{x}, \quad j^{2}=-1 \in \mathbb{C}^{*},
$$

with the maps

$$
\begin{gathered}
\phi: W_{\mathbb{R}} \rightarrow \operatorname{Gal}(\mathbb{C} / \mathbb{R}), \quad \phi(x)=1, \quad \phi(j x)=F r_{\infty}, \quad x \in \mathbb{C}, \\
r: \mathbb{R}^{*} \rightarrow W_{\mathbb{R}}^{a b}, \quad r(x)=x .
\end{gathered}
$$

Note that $W_{\mathbb{R}}$ is non-abelian and its abelianization is $W_{\mathbb{R}}^{a b}=W_{\mathbb{R}} /\left[W_{\mathbb{R}}, W_{\mathbb{R}}\right]=$ $\mathbb{R}^{*}$, where we identify $\operatorname{Gal}(\mathbb{C} / \mathbb{R})=\{ \pm 1\}$. Note that for Archimedean places thus defined Weil group coincides with the Weil-Deligne group.

Let ${ }^{L} G$ be a Langlands group dual to the reductive algebraic group $G$ used in the automorphic construction of $L$-factors above. Now with any continuous homomorphism of the Weil group in ${ }^{L} G$ and a finite-dimensional representation of ${ }^{L} G$ such that the image of the Weil group is semisimple one can associate an $L$-factor. Let us fix a complex finite-dimensional representation $\rho_{V}:{ }^{L} G \rightarrow G L(V, \mathbb{C})$ and consider its composition with a homomorphism $\pi_{p}: W_{\mathbb{Q}_{p}} \rightarrow{ }^{L} G$ such that the image of the inertia group is trivial. 
Then the corresponding local non-Archimedean $L$-factor is given by

$$
L_{p}\left(s, \pi, \rho_{V}\right)=\operatorname{det}_{V}\left(1-\rho_{V}\left(g_{p}\right) p^{-s}\right)^{-1}
$$

and coincides with the one defined using the local Hecke algebra (1.1) under appropriate identification of the parameters.

For Archimedean fields we consider the special case when the image of the Weil group under a homomorphism $W \rightarrow{ }^{L} G$ is abelian and the compact subgroup is in the kernel. Thus in the case of $F=\mathbb{C}$, we have the multiplicative group $\mathbb{R}_{>0}$ and in the case of $F=\mathbb{R}$ we have $\mathbb{R}^{*}=\mathbb{R}_{>0} \times\{ \pm 1\}$. The corresponding local Archimedean $L$-factors are given by

$$
\begin{gathered}
L_{\mathbb{C}}\left(s, \phi, \rho_{V}\right)=\operatorname{det}_{V}(2 \pi)^{-(s-\Lambda)} \Gamma(s-\Lambda), \\
L_{\mathbb{R}}\left(s, \phi, \rho_{V}, F r_{\infty}=+1\right)=\operatorname{det}_{V} \pi^{-\frac{s-\Lambda}{2}} \Gamma\left(\frac{s-\Lambda}{2}\right), \\
L_{\mathbb{R}}\left(s, \phi, \rho_{V}, F r_{\infty}=-1\right)=\operatorname{det}_{V} \pi^{-\frac{s-\Lambda}{2}} \Gamma\left(\frac{s+1-\Lambda}{2}\right),
\end{gathered}
$$

where $\Lambda$ is an image of the generator of $\mathbb{R}_{>0}$ and $F r_{\infty}= \pm 1$ describes the action of the Frobenius homomorphism $F r_{\infty} \in \operatorname{Gal}(\mathbb{C} / \mathbb{R})$ in $V$.

Remark 1.1. Local Archimedean $L$ factors are introduced in such a way that the completed global $L$ function (1.4) satisfies the functional Equation (1.5). This leaves a freedom to multiply the local Archimedean $L$-factor by a function of the form $A B^{s}, A \in \mathbb{C}^{*}, B \in \mathbb{R}_{>0}$ and is compensated by a freedom to redefine $\epsilon$-factor in (1.5).

\section{2. $\Gamma$-function via $2 d$ topological field theory}

In this section, we provide a functional integral representation of a product of $\Gamma$-functions using the Feynman path integral formulation of a twodimensional topological field theory. This leads to a functional integral representation of Archimedean $L$-factors. The two-dimensional theory involved is $S^{1} \times U_{\ell+1^{-}}$-equivariant version of the type A topological sigma model on the disk $D$ with the target space $V=\mathbb{C}^{\ell+1}$. Here $U_{\ell+1}$ acts on $\mathbb{C}^{\ell+1}$ via standard representation and $S^{1}$ acts by rotations $\alpha: z \rightarrow z \mathrm{e}^{\imath \alpha}$ on the disk $D=\{z \in \mathbb{C}|| z \mid \leq 1\}$. As a background material for two-dimensional topological field theories and functional integration of Gaussian measures over linear superspaces see e.g. [39]. 


\subsection{Type $A$ Topological sigma-models}

We start by recalling the standard construction of a topological sigma model associated with a Kähler manifold with non-negative first Chern class. For general discussion of the two-dimensional topological sigma models see $[4,11,45,46]$ and reference therein.

Let $X$ be a compact Kähler manifold of a complex dimension $(\ell+1)$ and $\mathcal{M}(\Sigma, X)=\operatorname{Map}(\Sigma, X)$ be the space of maps $\Phi: \Sigma \rightarrow X$ of a compact Riemann surface $\Sigma$ to $X$. Let $(z, \bar{z})$ be local complex coordinates on $\Sigma$. We pick a Hermitian metric $h$ on $\Sigma$ and denote $\sqrt{h} d^{2} z$ the corresponding measure on $\Sigma$. The complex structure defines a decomposition $d=\partial+\bar{\partial}$, $\partial=d z \partial_{z}, \bar{\partial}=d \bar{z} \partial_{\bar{z}}$ of the differential $d$ acting on the differential forms on $\Sigma$. Let $K$ and $\bar{K}$ be canonical and anti-canonical bundles over $\Sigma$. Let $\omega$ and $g$ be a Kähler form and the Kähler metric on $X$ and $T_{\mathbb{C}} X=T^{1,0} X \oplus T^{0,1} X$ be a decomposition of the complexified tangent bundle of $X$. We denote local complex coordinates on $X$ by $\left(\varphi^{j}, \bar{\varphi}^{\bar{j}}\right)$. Locally Levi-Civita connection $\Gamma$ and the corresponding Riemann curvature tensor $R$ are given by

$$
\Gamma_{j k}^{i}=g^{i \bar{n}} \partial_{j} g_{k \bar{n}}, \quad R_{i \bar{j} k \bar{l}}=g_{m \bar{j}} \partial_{\bar{l}} \Gamma_{i k}^{m} .
$$

Now let us specify the field content of a type A topological sigma model. We define commuting fields $F$ and $\bar{F}$ as sections of $K \otimes \Phi^{*}\left(T^{0,1}\right)$ and of $\bar{K} \otimes \Phi^{*}\left(T^{1,0}\right)$ correspondingly. The anticommuting fields $\chi, \bar{\chi}$ are sections of the bundles $\Phi^{*}\left(\Pi T^{1,0} X\right), \Phi^{*}\left(\Pi T^{0,1}\right)$ and anticommuting fields $\psi, \bar{\psi}$ are sections of the bundles $K \otimes \Phi^{*}\left(\Pi T^{0,1}\right), \bar{K} \otimes \Phi^{*}\left(\Pi T^{1,0}\right)$. Here $\Pi \mathcal{E}$ denotes the vector bundle $\mathcal{E}$ with the reverse parity of the fibers. Metrics $g$ on $X$ and $h$ on $\Sigma$ induce a Hermitian paring $\langle$,$\rangle on the space of sections of the$ considered bundles. Thus for example in local coordinates we have

$$
\langle\chi, \chi\rangle=\sum_{j=1}^{\ell+1} g_{i \bar{j}} \bar{\chi}^{\bar{j}} \chi^{i}, \quad\langle F, F\rangle=\sum_{j=1}^{\ell+1} h^{z \bar{z}} g_{i \bar{j}} \bar{F}_{z}^{\bar{j}} F_{\bar{z}}^{i} .
$$

The action functional of the type A topological sigma model is given by

$$
\begin{gathered}
S_{\Sigma}\left(t, t^{*}\right)=S_{0}(t)+S_{\text {top }}\left(t^{*}\right), \\
S_{0}(t)=\int_{\Sigma} d^{2} z \sqrt{h}(\imath\langle\mathcal{F}, \bar{\partial} \varphi\rangle+\imath\langle\overline{\mathcal{F}}, \partial \bar{\varphi}\rangle+\imath\langle\bar{\psi}, D \bar{\chi}\rangle \\
+\imath\langle\psi, \bar{D} \chi\rangle+t\langle\mathcal{F} \mathcal{F}\rangle+t\langle\bar{\psi}, R(\psi, \bar{\chi}) \chi\rangle), \\
S_{\text {top }}=t^{*} \int_{\Sigma} \Phi^{*}(\omega),
\end{gathered}
$$


where $\Phi^{*}(\omega)$ is a pull back of the Kähler form $\omega$ on $X$ along the map $\Phi: \Sigma \rightarrow X$. The fields $\mathcal{F}, \overline{\mathcal{F}}$ and the derivatives $D_{z}, \bar{D}_{\bar{z}}$ are locally given by

$$
\begin{gathered}
\mathcal{F}_{\bar{z}}^{j}=F_{\bar{z}}^{j}+\Gamma_{k l}^{j} \chi^{k} \psi_{\bar{z}}^{l}, \quad \mathcal{F}_{z}^{\bar{j}}=F_{z}^{\bar{j}}+\Gamma_{\bar{k} \bar{l}}^{\bar{j}} \chi^{\bar{k}} \psi_{z}^{\bar{l}}, \\
\left(D_{z} \bar{\chi}\right)^{\bar{j}}=\partial_{z} \bar{\chi}^{\bar{j}}+\Gamma_{\bar{k} \bar{l}}^{\bar{j}}(\varphi) \partial_{z} \bar{\varphi}^{\bar{k}} \bar{\chi}^{\bar{l}}, \quad\left(\bar{D}_{\bar{z}} \chi\right)^{j}=\partial_{\bar{z}} \chi^{j}+\Gamma_{k l}^{j}(\varphi) \partial_{\bar{z}} \varphi^{k} \chi^{l} .
\end{gathered}
$$

Here and in the following we imply the summation over repeating indexes.

The action functional (2.3) is invariant with respect to the transformations $A \rightarrow A+\epsilon \delta A$, where $\epsilon$ is an anticommuting parameter and the action of $\delta$ on the fields is

$$
\begin{aligned}
& \delta \varphi=\chi, \quad \delta \chi=0, \quad \delta \bar{\psi}=\bar{F}, \quad \delta \bar{F}=0, \\
& \delta \bar{\varphi}=\bar{\chi}, \quad \delta \bar{\chi}=0, \quad \delta \psi=F, \quad \delta F=0,
\end{aligned}
$$

and we have

$$
\delta^{2}=0
$$

The action $S_{0}(t)$ in $(2.3)$ can be written in the following form:

$$
S_{0}(t)=\int_{\Sigma} d^{2} z \sqrt{h} \delta \mathcal{V}(t),
$$

where

$$
\mathcal{V}(t)=\left\langle\psi, \frac{1}{2} t \mathcal{F}+\imath \bar{\partial} \varphi\right\rangle+\left\langle\bar{\psi}, \frac{1}{2} t \overline{\mathcal{F}}+\imath \partial \bar{\varphi}\right\rangle .
$$

The action (2.3) is obviously $\delta$-invariant. Indeed, $S_{0}(t)$ is $\delta$-invariant due to (2.9) and (2.8) and the second term $S_{\text {top }}$ is a topological invariant equal to the degree of the map $\Phi: \Sigma \rightarrow X$ and thus invariant under arbitrary infinitesimal deformations of the fields.

The transformation (2.7) can be interpreted as an action of the de Rham differential in the infinite-dimensional setting. Consider an odd vector bundle $\mathcal{E} \rightarrow \mathcal{M}(\Sigma, X)$ over the space $\mathcal{M}(\Sigma, X)$ of maps $\Phi: \Sigma \rightarrow X$ with a fiber isomorphic to the direct sum $\left(K \otimes \Phi^{*}\left(T^{0,1} X\right)\right) \oplus\left(\bar{K} \otimes \Phi^{*}\left(T^{1,0} X\right)\right)$. Then (2.7) defines the action of $\delta$, which coincides with action of the de Rham differential on differential forms on the infinite-dimensional supermanifold $\mathcal{E}$, where we imply $\chi:=\delta \varphi$ and $F:=\delta \psi$.

In the topological fields theories, we are interested in calculating the Feynman path integrals with the action (2.3) of $\delta$-closed functionals $A$ of 
the fields $\varphi, \bar{\varphi}, F, \bar{F}, \psi, \bar{\psi}, \chi, \bar{\chi}$

$$
\begin{aligned}
\langle A\rangle_{\Sigma}= & \int[\mathcal{D} \varphi][\mathcal{D} \bar{\varphi}][\mathcal{D} F][\mathcal{D} \bar{F}][\mathcal{D} \psi][\mathcal{D} \bar{\psi}][\mathcal{D} \chi][\mathcal{D} \bar{\chi}] e^{-S_{\Sigma}\left(t, t^{*}\right)} \\
& \times A(\varphi, \bar{\varphi}, F, \bar{F}, \psi, \bar{\psi}, \chi, \bar{\chi})
\end{aligned}
$$

In general, a functional integral is mathematically not well-defined and is usually handled using the standard renormalization routine of quantum field theory. In the following, we consider particular correlation functions in a special kind of type A topological sigma model when all calculations are mathematically well defined. Here we proceed formally implying that the integral (2.10) is well defined.

We would like to consider only correlation functions (2.10) of (the product of) observables $A$ which are $\delta$-closed. Then $\delta$-invariance of the action functional and the integration measure guarantees that the addition of a $\delta$-exact term to a functional $A$ does not change the correlation function $\langle A\rangle$. This allows us to identify the space of observables with $\delta$-cohomology. Local observables, i.e., depending on the values of the fields in the vicinity of a given point $(z, \bar{z})$ can be described explicitly in terms of the cohomology ring $H^{*}(X)$. One has canonical evaluation map

$$
e v: \Sigma \times \mathcal{M}(\Sigma, X) \rightarrow X
$$

Then for any $\beta \in H^{*}(X)$ we have $e v^{*}(\beta) \in H^{*}(\Sigma \times \mathcal{M}(\Sigma, X))=H^{*}(\Sigma) \otimes$ $H^{*}(\mathcal{M}(\Sigma, X))$. Let $e v^{*}(\beta)=\mathcal{O}_{\beta}^{(0)}+\mathcal{O}_{\beta}^{(1)}+\mathcal{O}_{\beta}^{(2)}$ be a decomposition of $e v^{*}$ $(\beta)$ with respect to the grading on the space of the differential forms over $\Sigma$. Then the condition

$$
(d+\delta)\left(\mathcal{O}_{\beta}^{(0)}+\mathcal{O}_{\beta}^{(1)}+\mathcal{O}_{\beta}^{(2)}\right)=0
$$

implies that the function $\mathcal{O}_{\beta}^{(0)}$ on $\Sigma$ is $\delta$-closed and its evaluation at any $p \in \Sigma$ gives a cohomology class in $H^{*}(\mathcal{M}(\Sigma, X))$. Moreover due to relation (2.12) we have

$$
d \mathcal{O}_{\beta}^{(0)}=-\delta \mathcal{O}_{\beta}^{(1)}
$$

and therefore this cohomology class in $H^{*}(\mathcal{M}(\Sigma, X))$ does not depend on the choice of the point $p \in \Sigma$. 
For example, the local observable at $p \in \Sigma$ constructed using the Kähler form

$$
\omega=\sum_{i, \bar{j}=1}^{\ell+1} \omega_{i \bar{j}} d \varphi^{j} \wedge d \bar{\varphi}^{\bar{j}}
$$

on $X$ is given by

$$
\mathcal{O}_{\omega}^{(0)}(p)=\sum_{i, \bar{j}=1}^{\ell+1} \omega_{i \bar{j}}(\varphi(p)) \chi^{i}(p) \bar{\chi}^{\bar{j}}(p)
$$

The corresponding differential form on $\mathcal{M}(\Sigma, X)$ provides a Kähler form on $\mathcal{M}(\Sigma, X)$.

Correlation functions in topological sigma models are closely related to GW invariants counting holomorphic curves on symplectic manifolds. This can be illustrated as follows. Taking $t=0$ in (2.3) we obtain the action linearly dependent on the fields $F, \bar{F}$. Functional integration over $F, \bar{F}$ in (2.10) reduces to the integration over the subset of holomorphic maps $\Phi: \Sigma \rightarrow X$. Thus the correlation functions in the topological sigma model at $t=0$ reduce to counting of holomorphic curves in $X$. Let us notice that although variation of the parameter $t$ in (2.3) changes the action by $\delta$-exact term the correlation functions of $\delta$-closed functionals depend on $t$ due to holomorphic anomaly [7]. Thus the interpretation in terms of holomorphic curves counting in general is not directly applicable for $t \neq 0$.

\subsection{Equivariant topological linear sigma model}

In the following, we need a simple generalization of the standard notion of the topological sigma-model discussed above. We consider equivariant version of type A topological linear sigma model on a disk $D$ with a noncompact target space. In the related context, the mathematical description of correlation functions in equivariant topological sigma models for compact $X$ and compact $\Sigma$ was considered by Givental [27-29]. For various application of equivariant cohomology in topological field theory, see e.g. [11] and reference therein.

Let us consider topological sigma model on the disk $D=\{z|| z \mid \leq 1\}$ with the target space $X=\mathbb{C}^{\ell+1}$ supplied with the Kähler form and Kähler metric

$$
\omega=\frac{\imath}{2} \sum_{j=1}^{\ell+1} d \varphi^{j} \wedge d \bar{\varphi}^{j}, \quad g=\frac{1}{2} \sum_{j=1}^{\ell+1}\left(d \varphi^{j} \otimes d \bar{\varphi}^{j}+d \bar{\varphi}^{j} \otimes d \varphi^{j}\right)
$$


We fix the flat metric $h$ on $D$

$$
h=\frac{1}{2}(d z d \bar{z}+d \bar{z} d z)=(d r)^{2}+r^{2}(d \sigma)^{2}, \quad r \in[0,1], \quad \sigma \in[0,2 \pi]
$$

where $z=r e^{\imath \sigma}$.

The action (2.3) for $X=\mathbb{C}^{\ell+1}$ and $t=t^{*}=0$ is given by

$$
S_{D}=\int_{D} d^{2} z \delta \mathcal{V}=\imath \int_{D} d^{2} z(\langle F, \bar{\partial} \varphi\rangle+\langle\bar{F}, \partial \bar{\varphi}\rangle+\langle\bar{\psi}, \partial \bar{\chi}\rangle+\langle\psi, \bar{\partial} \chi\rangle)
$$

where $\mathcal{V}=\imath\langle\psi, \bar{\partial} \varphi\rangle+\imath\langle\bar{\psi}, \partial \bar{\varphi}\rangle$ and

$$
\begin{aligned}
& \delta \varphi=\chi, \quad \delta \chi=0, \quad \delta \bar{\psi}=\bar{F}, \quad \delta \bar{F}=0, \\
& \delta \bar{\varphi}=\bar{\chi}, \quad \delta \bar{\chi}=0, \quad \delta \psi=F, \quad \delta F=0 .
\end{aligned}
$$

To construct an equivariant extension of the topological theory with the action (2.18) we modify transformations (2.19) following the interpretation of $\delta$ as a de Rham differential in the infinite-dimensional setting. Let us first recall the standard construction of an algebraic model of equivariant cohomology. Let $M$ be a $2(\ell+1)$-dimensional manifold supplied with an action of a compact Lie group $G$. Let $\mathfrak{g}$ be the corresponding Lie algebra and $\mathfrak{g}^{*}$ be its dual. Pick a bases $\left\{t^{a}\right\}, a=1, \ldots$, dim $\mathfrak{g}$ in the Lie algebra $\mathfrak{g}$ and let $\left\{v^{a}\right\}$ be the set of the corresponding vector fields on $M$. Introduce a bases $\left\{u^{a}\right\}, a=1, \ldots, \operatorname{dim} \mathfrak{g}$ in $\mathfrak{g}^{*}$ dual to the bases $\left\{t^{a}\right\} a=1, \ldots, \operatorname{dim} \mathfrak{g}$. The Cartan algebraic model of $G$-equivariant de Rham cohomology $H_{G}^{*}(M)$ is defined as a cohomology of the following equivariant extension $\left(\Omega_{G}^{*}(M), d_{G}\right)$ of the standard de Rham complex $\left(\Omega^{*}(M), d\right)$

$$
\Omega_{G}^{*}(M)=\left(\Omega^{*}(M) \otimes S^{*}\left[\mathfrak{g}^{*}\right]\right)^{G}, \quad d_{G}=d-\sum_{a=1} u_{a} i_{v^{a}}
$$

In (2.20) the standard coadjoint action of $G$ on $\mathfrak{g}^{*}$ is implied. We have

$$
d_{G}^{2}=-\mathcal{L}_{\sum_{a=1}^{\operatorname{dim} \mathfrak{g}} u_{a} v^{a}}, \quad \mathcal{L}_{v}=d i_{v}+i_{v} d
$$

where $\mathcal{L}_{v}$ is the Lie derivative along a vector field $v$. Note that the equivariant differential $d_{G}$ satisfies $d_{G}^{2}=0$ when acting on $\Omega_{G}^{*}(M)$. The cohomology groups $H_{G}^{*}(M)$ of the complex (2.20) naturally have a structure of modules over $H_{G}^{*}(\mathrm{pt})=\left(S^{*}\left(\mathfrak{g}^{*}\right)\right)^{G}$ and the algebra $\left(S^{*}\left(\mathfrak{g}^{*}\right)\right)^{G}$ of $G$ invariant polynomial functions on $\mathfrak{g}$ can be identified with the algebra $\left(S^{*}\left(\mathfrak{h}^{*}\right)\right)^{W}$ of Weyl-invariant functions on the Cartan subalgebra $\mathfrak{h} \subset \mathfrak{g}$. 
We have the standard action of $U_{\ell+1}$ on $V=\mathbb{C}^{\ell+1}$ and an action of $S^{1}$ on $D$ by rotations $\sigma \rightarrow \sigma+\alpha$ leaving the metric (2.17) invariant. The action of $G=S^{1} \times U_{\ell+1}$ lifts naturally to the action on the fields $(F, \bar{F}, \varphi, \bar{\varphi}, \psi, \bar{\psi}$, $\chi, \bar{\chi})$. For instance the infinitesimal rotation acts via the Lie derivative $\mathcal{L}_{v_{0}}$ along the vector field $v_{0}=\frac{\partial}{\partial \sigma}$

$$
\delta_{v_{0}} \varphi^{j}=\mathcal{L}_{v_{0}} \varphi^{j}=i_{v_{0}} d \varphi^{j}, \quad \delta_{v_{0}} F^{j}=\mathcal{L}_{v_{0}} F^{j}=i_{v_{0}} d F^{j}+d\left(i_{v_{0}} F^{j}\right) .
$$

Let $\hbar v_{0}$ be an element of the Lie algebra of $S^{1}$ and $\Lambda$ be an image of the element $\sum_{a=1}^{\operatorname{dim} \mathfrak{g}} u_{a} t^{a}$ of the Lie algebra $\mathfrak{u}_{\ell+1}=\operatorname{Lie}\left(U_{\ell+1}\right)$ in the representation $V=\mathbb{C}^{\ell+1}$. The $G$-equivariant analog $\delta_{G}$ of the transformation $\delta(2.19)$ is obtained adapting (2.20) to the considered action of $G=S^{1} \times U_{\ell+1}$ on the fields of topological sigma model

$$
\begin{aligned}
& \delta_{G} \varphi=\chi, \quad \delta_{G} \chi=-\left(\imath \Lambda \varphi+\hbar \mathcal{L}_{v_{0}} \varphi\right), \quad \delta_{G} \psi=F, \\
& \delta_{G} F=-\left(\imath \Lambda \psi+\hbar \mathcal{L}_{v_{0}} \psi\right), \\
& \delta_{G} \bar{\varphi}=\bar{\chi}, \quad \delta_{G} \bar{\chi}=-\left(-\imath \Lambda \bar{\varphi}+\hbar \mathcal{L}_{v_{0}} \bar{\varphi}\right), \quad \delta_{G} \bar{\psi}=\bar{F}, \\
& \delta_{G} \bar{F}=-\left(-\imath \Lambda \bar{\psi}+\hbar \mathcal{L}_{v_{0}} \bar{\psi}\right) .
\end{aligned}
$$

It is easy to check that the action $(2.18)$ is both $G$ - and $\delta_{G}$-invariant.

Equivariant observables are given by $\delta_{G}$-closed $G$-invariant functionals of the fields. The simple direct check shows that the following functional defines an equivariant extension of the observable (2.15) corresponding to the Kähler form (2.16)

$$
\mathcal{O}_{\Lambda, \hbar}(r)=\frac{\imath}{2} \int_{0}^{2 \pi} d \sigma\left(-\left\langle\chi\left(r \mathrm{e}^{\imath \sigma}\right), \chi\left(r \mathrm{e}^{\imath \sigma}\right)\right\rangle+\left\langle\varphi\left(r \mathrm{e}^{\imath \sigma}\right),\left(\imath \Lambda+\hbar \mathcal{L}_{v_{0}}\right) \varphi\left(r \mathrm{e}^{\imath \sigma}\right)\right\rangle\right) .
$$

We would like to consider the functional integral over the disk with the action (2.18) deformed by the observable $\mathcal{O}_{\Lambda, \hbar}:=\mathcal{O}_{\Lambda, \hbar}(r=1)$, where $\mathcal{O}_{\Lambda, \hbar}(r)$ is given by $(2.24)$. Note that this functional integral is a Gaussian integral in the infinite-dimensional space and thus allows mathematically correct formulation using for instance the $\zeta$-function regularization $[32,38]$.

Theorem 2.1. Let $V=\mathbb{C}^{\ell+1}$ be the standard representation of $U_{\ell+1}$. Let $\Lambda$ be the image of an element $u \in \mathfrak{u}_{\ell+1}$ in $\operatorname{End}(V)$. Then the following identity holds

$$
\left\langle\mathrm{e}^{\mu \mathcal{O}_{\Lambda, \hbar}}\right\rangle_{D}=\hbar^{-\frac{\ell+1}{2}} \operatorname{det}_{V}\left(\frac{2}{\mu \hbar}\right)^{-\Lambda / \hbar} \Gamma(\Lambda / \hbar)
$$


where $\mathcal{O}_{\Lambda, \hbar}$ is given by (2.24) for $r=1$. The functional integral in the $S^{1} \times U_{\ell+1}$-equivariant Type $A$ topological linear sigma model with the target space $V=\mathbb{C}^{\ell+1}$ and the action functional (2.18) is calculated using $\zeta$ function regularization. After taking $\mu=2 / \pi, \hbar=1$ and making the change of variables $\Lambda \rightarrow(s \cdot \mathrm{id}-\Lambda) / 2$ the partition function (2.25) turns into local Archimedean L-factor (1.11).

Proof. The functional integral in (2.25) regularized using $\zeta$-function regularization is invariant with respect to the action of $U_{\ell+1}$. Thus the correlation function $(2.25)$ is a central function of $(\ell+1) \times(\ell+1)$ matrix $\Lambda$ and can be reconstructed from its restriction to diagonal matrices $\Lambda=$ $\operatorname{diag}\left(\lambda_{1}, \ldots, \lambda_{\ell+1}\right)$. Then the integral is factorized on the product of the functional integrals in $S^{1} \times U_{1}$-topological sigma models with one-dimensional target spaces $X=\mathbb{C}$. Using the Proposition 2.1 proved in the next subsection we have

$$
\left\langle\mathrm{e}^{\mu \mathcal{O}_{\Lambda, \hbar}}\right\rangle_{D}=\hbar^{-\frac{\ell+1}{2}} \prod_{j=1}^{\ell+1}\left(\frac{2}{\mu \hbar}\right)^{-\lambda_{j} / \hbar} \Gamma\left(\lambda_{j} / \hbar\right), \quad \Lambda=\operatorname{diag}\left(\lambda_{1}, \ldots, \lambda_{\ell+1}\right) .
$$

Now it is clear that the r.h.s. of (2.26) is a restriction of r.h.s. of (2.25) to the diagonal matrix $\Lambda=\operatorname{diag}\left(\lambda_{1}, \ldots, \lambda_{\ell+1}\right)$

Remark 2.1. According to Remark 1.1, the correlation function (2.25) for arbitrary $\mu$ and $\hbar$ can also be considered as a complex Archimedean $L$-factor. The $\mu$-dependence of the functional integral defines also a renormalization scale dependence (see e.g. [32]). For example using another regularization of the Gaussian functional integral (i.e., different form of $\zeta$-function regularization) we would obtain the same answer up to a multiplication by a factor of the form $A B^{s}$.

\subsection{Calculation of the functional integral}

In this subsection, we prove (2.26). It is enough to consider the case of $\ell=0$.

Proposition 2.1. The following integral representation for $\Gamma$-function holds

$$
\Gamma(\lambda / \hbar)=\hbar^{\frac{1}{2}}\left(\frac{2}{\mu \hbar}\right)^{\lambda / \hbar}\left\langle\mathrm{e}^{\mu \mathcal{O}_{\lambda, \hbar}}\right\rangle_{D}
$$


where

$$
\mathcal{O}_{\lambda, \hbar}=\frac{\imath}{2} \int_{\partial D=S^{1}} d \sigma\left(-\bar{\chi} \chi+\bar{\varphi}\left(\imath \lambda+\hbar \partial_{\sigma}\right) \varphi\right),
$$

and the functional integral is taken with the action functional

$$
S_{D}=\imath \int_{D} d^{2} z\left(\bar{F}_{z} \partial_{\bar{z}} \varphi+F_{\bar{z}} \partial_{z} \bar{\varphi}+\psi_{\bar{z}} \partial_{z} \bar{\chi}+\bar{\psi}_{z} \partial_{\bar{z}} \chi\right) .
$$

Proof. Decompose the fields $\varphi$ and $\chi$

$$
\varphi=\varphi_{0}+\varphi_{h}, \quad \chi=\chi_{0}+\chi_{h}
$$

so that $\varphi_{0}$ and $\chi_{0}$ satisfy the boundary conditions

$$
\left.\varphi_{0}\right|_{\partial D=S^{1}}=0,\left.\quad \chi_{0}\right|_{\partial D=S^{1}}=0,
$$

and $\varphi_{h}, \chi_{h}$ are harmonic functions

$$
\partial_{z} \partial_{\bar{z}} \varphi_{h}=0, \quad \partial_{z} \partial_{\bar{z}} \chi_{h}=0
$$

Let us also decompose the fields $F$ and $\psi$ as follows:

$$
\begin{aligned}
& \bar{F}_{z} d z=\bar{G}_{z} d z+\bar{f}_{z} d z, \quad F_{\bar{z}} d \bar{z}=G_{\bar{z}} d \bar{z}+f_{\bar{z}} d \bar{z} \\
& \bar{\psi}_{z} d z=\bar{\xi}_{z} d z+\bar{\rho}_{z} d z, \quad \psi_{\bar{z}} d \bar{z}=\xi_{\bar{z}} d \bar{z}+\rho_{\bar{z}} d \bar{z}
\end{aligned}
$$

where one-forms $f, \rho$ and $\bar{f}, \bar{\rho}$ satisfy the equations

$$
\partial_{\bar{z}} \bar{f}_{z}=0, \quad \partial_{z} f_{\bar{z}}=0, \quad \partial_{\bar{z}} \bar{\rho}_{z}=0, \quad \partial_{z} \rho_{\bar{z}}=0
$$

The fields $G, \bar{G}$ should be in the subspace orthogonal to the subspace spanned by $f$ and $\bar{f}$ satisfying (2.33)

$$
\langle\bar{G}, f\rangle=\int_{D} d^{2} z \bar{G}_{z} f_{\bar{z}}=0, \quad\langle G, \bar{f}\rangle=\int_{D} d^{2} z G_{\bar{z}} \bar{f}_{z}=0 .
$$

We impose similarly conditions on $\xi$ and $\bar{\xi}$. The following identity can be easily checked

$$
\begin{aligned}
\int_{D} d^{2} z\left(\bar{F}_{z} \partial_{\bar{z}} \varphi+F_{\bar{z}} \partial_{z} \bar{\varphi}\right)= & \int_{D} d^{2} z\left(\bar{G}_{z} \partial_{\bar{z}} \varphi_{0}+G_{\bar{z}} \partial_{z} \bar{\varphi}_{0}\right) \\
& +\int_{\partial D}\left(d z \bar{f}_{z} \varphi_{h}-d \bar{z} f_{\bar{z}} \bar{\varphi}_{h}\right)
\end{aligned}
$$


Using (2.35) we have the decomposition of the action (2.29)

$$
S_{D}=S_{\mathrm{bulk}}+S_{\mathrm{boundary}}
$$

where

$$
S_{\text {bulk }}=\imath \int_{D} d^{2} z\left(\bar{G}_{z} \partial_{\bar{z}} \varphi_{0}+G_{\bar{z}} \partial_{z} \bar{\varphi}_{0}+\bar{\xi}_{z} \partial_{\bar{z}} \chi_{0}+\xi_{\bar{z}} \partial_{z} \bar{\chi}_{0}\right)
$$

and

$$
S_{\text {boundary }}=\imath \int_{S^{1}=\partial D} d \sigma\left(\bar{f}_{z} \varphi_{h}-f_{\bar{z}} \bar{\varphi}_{h}+\bar{\rho}_{z} \chi_{h}-\rho_{\bar{z}} \bar{\chi}_{h}\right) \text {. }
$$

The integration measure is defined using the standard Hermitian metric on tensor fields on the disk. For example, the measure $[D \varphi][D \bar{\varphi}]$ over $(\varphi, \bar{\varphi})$ is induced by the metric

$$
\|\delta \varphi\|^{2}=\int_{D} d^{2} z \delta \bar{\varphi} \delta \varphi, \quad\|\delta \chi\|^{2}=\int_{D} d^{2} z \delta \bar{\chi} \delta \chi
$$

Taking into account that super-manifolds have canonical integration measure (see the Appendix) the integration measures can be split as follows:

$$
\begin{aligned}
{[D F][D \bar{F}][D \psi][D \bar{\psi}]=} & {[D G][D \bar{G}][D f][D \bar{f}][D \xi][D \bar{\xi}][D \rho][D \bar{\rho}] } \\
{[D \varphi][D \bar{\varphi}][D \chi][D \bar{\chi}]=} & {\left[D \varphi_{0}\right]\left[D \bar{\varphi}_{0}\right]\left[D \varphi_{h}\right]\left[D \bar{\varphi}_{h}\right]\left[D \chi_{0}\right]\left[D \bar{\chi}_{0}\right] } \\
& \times\left[D \chi_{h}\right]\left[D \bar{\chi}_{h}\right]
\end{aligned}
$$

Thus using the decomposition (2.36) we have a factorization of the functional integral in (2.27) into the product of the integrals over $\left(G, \varphi_{0}, \xi, \chi_{0}\right)$ and $\left(f, \varphi_{h}, \rho, \chi_{h}\right)$. Consider first the integration over $\left(G, \varphi_{0}, \xi, \chi_{0}\right)$ with the action

$$
S_{\mathrm{bulk}}=\imath \int d^{2} z\left(\bar{G}_{z} \partial_{\bar{z}} \varphi_{0}+G_{\bar{z}} \partial_{z} \bar{\varphi}_{0}+\bar{\xi}_{z} \partial_{\bar{z}} \chi_{0}+\xi_{\bar{z}} \partial_{z} \bar{\chi}_{0}\right)
$$

This is an infinite-dimensional analog of the integral (A.5) defined by $\zeta$ function regularization. The integral gives a trivial contribution to (2.27) (the corresponding integrals over odd and even variables cancel identically). Thus one should calculate the following functional integral:

$$
\int\left[\mathcal{D} \varphi_{h}\right]\left[\mathcal{D} \bar{\varphi}_{h}\right][\mathcal{D} f][\mathcal{D} \bar{f}][\mathcal{D} \rho][\mathcal{D} \bar{\rho}]\left[\mathcal{D} \chi_{h}\right]\left[\mathcal{D}{\overline{\chi_{h}}}\right] e^{-S_{*}}
$$


where

$$
\begin{aligned}
S_{*}= & \int_{S^{1}=\partial D} d \sigma\left(\imath \bar{f}_{z} \varphi_{h}-\imath f_{\bar{z}} \bar{\varphi}_{h}+\imath \bar{\rho}_{z} \chi_{h}-\imath \rho_{\bar{z}} \bar{\chi}_{h}\right. \\
& \left.+\mu \frac{\imath}{2}\left(\chi \bar{\chi}+\bar{\varphi}\left(\imath \lambda+\hbar \partial_{\sigma}\right) \varphi\right)\right) .
\end{aligned}
$$

Consider decomposition of the fields

$$
\begin{aligned}
\varphi_{h}(z, \bar{z}) & =\sum_{n>0} \varphi_{-n} \bar{z}^{n}+\sum_{n \geq 0} \varphi_{n} z^{n}=\varphi_{-}(\bar{z})+\varphi_{+}(z), \\
\bar{\varphi}_{h}(z, \bar{z}) & =\sum_{n \geq 0} \bar{\varphi}_{-n} \bar{z}^{n}+\sum_{n>0} \bar{\varphi}_{n} z^{n}=\bar{\varphi}_{-}(z)+\bar{\varphi}_{+}(\bar{z}) \\
\chi_{h}(z, \bar{z}) & =\sum_{n>0} \chi_{-n} \bar{z}^{n}+\sum_{n \geq 0} \chi_{n} z^{n}=\chi_{-}(\bar{z})+\chi_{+}(z), \\
\bar{\chi}_{h}(z, \bar{z}) & =\sum_{n \geq 0} \bar{\chi}_{-n} \bar{z}^{n}+\sum_{n>0} \bar{\chi}_{n} z^{n}=\bar{\chi}_{-}(z)+\bar{\chi}_{+}(\bar{z}) .
\end{aligned}
$$

Taking into account (2.33) one can write the action (2.44) as follows:

$$
\begin{aligned}
S_{*}= & \int_{S^{1}=\partial D} d \sigma\left(\imath \bar{f}_{z} \varphi_{-}-\imath f_{\bar{z}} \bar{\varphi}_{-}+\imath \bar{\rho}_{z} \chi_{-}-\imath \rho_{\bar{z}} \bar{\chi}_{-}\right. \\
& \left.+\mu \frac{\imath}{2}\left(\chi \bar{\chi}+\bar{\varphi}\left(\imath \lambda+\hbar \partial_{\sigma}\right) \varphi\right)\right) .
\end{aligned}
$$

Integrating over $\left(\varphi_{-}, \bar{\varphi}_{-}, f, \bar{f}\right)$ and $\left(\chi_{-}, \bar{\chi}_{-}, \rho, \bar{\rho}\right)$ and using (A.2) we are left with the following integral:

$$
\left\langle\mathrm{e}^{\mu \mathcal{O}_{\lambda, \hbar}}\right\rangle_{D}=\int\left[\mathcal{D} \varphi_{+}\right]\left[\mathcal{D} \bar{\varphi}_{+}\right]\left[\mathcal{D} \chi_{+}\right]\left[\mathcal{D}{\overline{\chi_{+}}}^{-} \mathrm{e}^{-S_{b}}\right.
$$

where

$$
S_{b}=-\mu \frac{\imath}{2} \int_{0}^{2 \pi} d \sigma\left(\chi_{+} \bar{\chi}_{+}+\bar{\varphi}_{+}\left(\imath \lambda+\hbar \partial_{\sigma}\right) \varphi_{+}\right) .
$$

and functional measure is defined using the metric induced by (2.39) on the space of the fields $\left(\varphi_{+}, \bar{\varphi}_{+}, \chi_{+}, \bar{\chi}_{+}\right)$.

The functional integral (2.47) can be easily calculated using the $\zeta$ function regularization. Using the normalization of the integration measure 
given in the appendix we have

$$
\left\langle\mathrm{e}^{\mu \mathcal{O}_{\lambda, \hbar}}\right\rangle_{D}=\frac{\operatorname{det} \mathcal{D}_{0}}{\operatorname{det} \mathcal{D}}
$$

Here the differential operator $\mathcal{D}=-\frac{\imath}{2} \mu\left(\hbar \frac{\partial}{\partial \sigma}+\imath \lambda\right)$ acts in the space of complex-valued functions on a circle $\sigma \sim \sigma+2 \pi$ which are restrictions of holomorphic functions on the disk $D$. The operator $\mathcal{D}_{0}$ acts by multiplication on $2 \pi \cdot \frac{\mu}{2}$. The spectrum of $\mathcal{D}$ is

$$
\lambda_{n}=\frac{\mu}{2}(\hbar n+\lambda), \quad n \geq 0
$$

and using (A.8) we have

$$
\ln \operatorname{det} \mathcal{D}=\left(\frac{1}{2}-\frac{\lambda}{\hbar}\right) \ln \left(\frac{\mu \hbar}{2}\right)+\frac{1}{2} \ln 2 \pi-\ln \Gamma\left(\frac{\lambda}{\hbar}\right) .
$$

The determinant of $\mathcal{D}_{0}$ is calculated using (A.9)

$$
\ln \operatorname{det} \mathcal{D}_{0}=\frac{1}{2} \ln \left(2 \pi \frac{\mu}{2}\right)
$$

Substitution of (2.51) and (2.52) into (2.49) gives (2.27)

\section{3. $\quad \Gamma$-function as an equivariant symplectic volume}

In the previous section, we represent a product of $\Gamma$-functions as a particular Gaussian functional integral. In this section, we interpret this functional integral as an equivariant symplectic volume of the space of holomorphic maps of the disk $D$ into $\mathbb{C}^{\ell+1}$. According to the general correspondence principle in quantum mechanics equivariant symplectic volume can be considered as a classical limit of a partition function of quantum system obtained by a quantization of the symplectic manifold. We apply this heuristic principle to the space of holomorphic maps of the disk $D \rightarrow \mathbb{C}^{\ell+1}$. The corresponding partition function is given by the product of $q$-deformed $\Gamma$-functions. In the next section, we provide a quantum field theory interpretation of this result by identifying the product of $q$-deformed $\Gamma$-functions with the correlation function in the three-dimensional equivariant topological linear sigma-model. 


\section{1. $U_{\ell+1}$-equivariant symplectic volume of $\mathbb{C}^{\ell+1}$}

In this subsection, we consider a calculation of $U_{\ell+1}$-equivariant symplectic volume of $\mathbb{C}^{\ell+1}$ and its interpretation as an assymptotic of a partition function of the associated quantum system. These considerations provide an example for the discussion of the equivariant symplectic volume of the space of holomorphic maps of the disk $D$ into $\mathbb{C}^{\ell+1}$.

Let $M$ be a $2(\ell+1)$-dimensional symplectic manifold with a symplectic form $\omega$. Let $G$ be a compact Lie group acting on $M$. Let $\mathfrak{g}^{*}$ be a dual to the Lie algebra $\mathfrak{g}$ of $G$. Let the action of $G$ on $(M, \omega)$ is Hamiltonian with the momentum map $H: M \rightarrow \mathfrak{g}^{*}$. Define $G$-equivariant symplectic volume of $M$ as follows

$$
Z(M, \lambda)=\int_{M} \mathrm{e}^{\langle\lambda, H\rangle+\omega}=\int_{M} \frac{\omega^{\ell+1}}{(\ell+1) !} \mathrm{e}^{\langle\lambda, H\rangle}, \quad \lambda \in \mathfrak{g},
$$

where $\langle$,$\rangle is the paring between \mathfrak{g}$ and its dual $\mathfrak{g}^{*}$.

The term "equivariant volume" for the integral (3.1) comes from the interpretation of $\omega_{G}:=\omega+\langle\lambda, H\rangle$ as a $G$-equivariant extension of the symplectic form $\omega$. Indeed, $\omega_{G}$ is a $G$-invariant $d_{G}$-closed two-form and thus defines an element in $H_{G}^{2}(M)$ using the Cartan model (2.20) of the equivariant cohomology. The interpretation of (3.1) in terms of equivariant cohomology leads to a possibility to apply a powerful localization technique to the calculation the integrals (3.1) (see [15] for a direct calculation and [5,47] for equivariant localization approach in abelian and non-abelian cases).

Integral expression (3.1) is invariant with respect to the adjoint action of $G$ on $\lambda \in \mathfrak{g}$ and thus can be uniquely reconstructed from the specialization of (3.1) to the case when $\lambda$ belongs to a Cartan subalgebra $\mathfrak{h} \subset \mathfrak{g}$

$$
Z(M, \underline{\lambda})=\int_{M} \mathrm{e}^{\omega+\sum_{j=1}^{\operatorname{dim} \mathfrak{h}} \lambda_{j} H_{j}}, \quad \underline{\lambda}=\left(\lambda_{1}, \lambda_{2}, \ldots, \lambda_{\operatorname{dim} \mathfrak{h}}\right) \in \mathfrak{h} .
$$

Let us consider a particular example of the equivariant symplectic volume calculation. Let $M=\mathbb{C}^{\ell+1}$ be supplied with the symplectic structure

$$
\omega=\frac{\imath}{2} \sum_{j=1}^{\ell+1} d z^{j} \wedge d \bar{z}^{j}
$$

The symplectic structure (3.3) is invariant with respect to the standard action of $U_{\ell+1}$ on $\mathbb{C}^{\ell+1}$. The momenta corresponding to the action of the 
generators of Cartan subgroup $\left(U_{1}\right)^{\ell+1} \subset U_{\ell+1}$ are given by

$$
H_{j}=-\frac{1}{2}\left|z^{j}\right|^{2}, \quad j=1, \ldots, \ell+1 .
$$

The $\left(U_{1}\right)^{\ell+1}$-equivariant extension $\omega_{\left(U_{1}\right)^{\ell+1}}$ of $\omega$ satisfies the condition

$$
\left(d-\sum_{j=1}^{\ell+1} \lambda_{j} i_{V_{j}}\right) \omega_{\left(U_{1}\right)^{\ell+1}}=0
$$

and is given by

$$
\omega_{U_{\ell+1}}=\omega+\sum_{j=1}^{\ell+1} \lambda_{j} H_{j}=\frac{\imath}{2} \sum_{j=1}^{\ell+1}\left(d z^{j} \wedge d \bar{z}^{j}+\imath \lambda_{j}\left|z^{j}\right|^{2}\right) .
$$

The simple direct calculation gives

$$
Z\left(\mathbb{C}^{\ell+1}, \underline{\lambda}\right)=\int_{\mathbb{C}^{\ell+1}} \mathrm{e}^{\omega+\sum_{j=1}^{\ell+1} \lambda_{j} H_{j}}=\int_{\mathbb{C}^{\ell+1}} \frac{\omega^{\ell+1}}{(\ell+1) !} \mathrm{e}^{\sum_{j=1}^{\ell+1} \lambda_{j} H_{j}}=\frac{(2 \pi)^{\ell+1}}{\prod_{j=1}^{\ell+1} \lambda_{j}},
$$

where $\underline{\lambda}=\left(\lambda_{1}, \lambda_{2}, \ldots, \lambda_{\ell+1}\right)$ is an element of the Cartan subalgebra of $\mathfrak{u}_{\ell+1}=$ $\operatorname{Lie}\left(U_{\ell+1}\right)$.

It is useful to rewrite the integral (3.7) using auxiliary anticommuting variables $\left(\eta^{j}, \bar{\eta}^{j}\right), j=1, \ldots,(\ell+1)$. The standard identification of polynomial differential forms on $\mathbb{C}^{\ell+1}$ with polynomials in commuting and anticommuting variables $\mathbb{C}\left[z^{j}, \bar{z}^{j}, \eta^{j}, \bar{\eta}^{j}\right]$, leads to the following expression for the symplectic form (3.3):

$$
\omega=\frac{\imath}{2} \sum_{j=1}^{\ell+1} \eta^{j} \bar{\eta}^{j}
$$

The integral (3.7) can be rewritten using the integration over anticommuting variables in the following form:

$$
\begin{aligned}
Z\left(\mathbb{C}^{\ell+1}, \underline{\lambda}\right) & =\int_{\mathbb{C}^{\ell+1 \mid \ell+1}} \prod_{j=1}^{\ell+1} d z^{j} d \bar{z}^{j} \prod_{j=1}^{\ell+1} d \eta^{j} d \bar{\eta}^{j} \mathrm{e}^{\frac{\imath \mu}{2} \sum_{j=1}^{\ell+1}\left(\eta^{j} \bar{\eta}^{j}+\imath \lambda_{j}\left|z^{j}\right|^{2}\right)} \\
& =\frac{(2 \pi)^{\ell+1}}{\prod_{j=1}^{\ell+1} \lambda_{j}}
\end{aligned}
$$

Here $\mathbb{C}^{\ell+1 \mid \ell+1}$ shall be considered as an odd tangent bundle $\Pi T \mathbb{C}^{\ell+1}$ to $\mathbb{C}^{\ell+1}$. Note that the integral is independent on $\mu$. 
According to the Correspondence Principle in quantum mechanics/ statistical mechanics the integral over symplectic manifolds of the form (3.1) describes an asymptotic of the partition function of the corresponding quantum system. More precisely, if a quantization of the symplectic manifold $(M, \omega)$ exists and $\hat{H}_{j}$ are quantum counterparts of the Hamiltonians $H_{j}$ then the equivariant volume (3.2) describes the asymptotic of the following trace over the Hilbert space of the corresponding quantum system:

$$
Z_{\tilde{\hbar}}(M, \beta, \underline{\lambda})=\operatorname{Tr}_{\mathcal{H}} \mathrm{e}^{-\frac{\beta}{\tilde{\hbar}} \sum_{j=1}^{\ell+1} \lambda_{j} \hat{H}_{j}},
$$

where $\tilde{\hbar}$ is parameter of quantum deformation.

For example in the case of $M=\mathbb{C}^{\ell+1}$ supplied with the symplectic form (3.3) the corresponding quantum system can be easily found. The quantum counterpart of the algebra of polynomial functions $\mathbb{C}\left[z^{1}, \ldots, z^{\ell+1}, \bar{z}^{1}, \ldots\right.$, $\left.\bar{z}^{\ell+1}\right]$ is a Heisenberg algebra generated by $\hat{z}^{1}, \ldots, \hat{z}^{\ell+1}, \hat{\bar{z}}^{1}, \ldots, \hat{\bar{z}}^{\ell+1}$ with the relations

$$
\left[\hat{z}^{i}, \hat{z}^{j}\right]=0, \quad\left[\hat{\bar{z}}^{i}, \hat{\bar{z}}^{j}\right]=0, \quad\left[\hat{\bar{z}}^{i}, \hat{z}^{j}\right]=-2 \tilde{\hbar} \delta^{i j}
$$

This algebra can be represented in the space of polynomials $\mathcal{V}^{(\ell+1)}=$ $\mathbb{C}\left[z^{1}, \ldots, z^{\ell+1}\right]$ with the action of the generators

$$
\hat{z}^{j}=z^{j}, \quad \hat{\bar{z}}^{j}=-2 \tilde{\hbar} \frac{\partial}{\partial z^{j}} .
$$

The space $\mathcal{V}^{(\ell+1)}$ has a natural structure of $U_{\ell+1}$-module and $U_{\ell+1}$-character of the module $\mathcal{V}^{(\ell+1)}$ is easy to calculate. The generators of the Cartan subalgebra of $\mathfrak{u}_{\ell+1}$ are realized by the following differential operators:

$$
\hat{H}_{j}=\tilde{\hbar} z^{j} \frac{\partial}{\partial z^{j}}, \quad j=1, \ldots, \ell+1
$$

and provide a quantization of the classical Hamiltonians (3.4). The character of $U_{\ell+1}$-module $\mathcal{V}^{(\ell+1)}$ is given by (3.10) and a simple calculation gives

$$
Z_{\tilde{\hbar}}\left(\mathbb{C}^{\ell+1}, \beta, \underline{\lambda}\right)=\operatorname{Tr}_{\mathcal{V}^{\ell+1}} \mathrm{e}^{-\frac{\beta}{\tilde{\hbar}} \sum_{j=1}^{\ell+1} \lambda_{j} \hat{H}_{j}}=\prod_{j=1}^{\ell+1} \frac{1}{1-\mathrm{e}^{-\beta \lambda_{j}}} .
$$

Then the following relation between equivariant volume (3.7) and the character (3.13) holds

$$
Z\left(\mathbb{C}^{\ell+1}, \underline{\lambda}\right)=\lim _{\beta \rightarrow 0}(2 \pi \beta)^{\ell+1} Z_{\tilde{\hbar}}\left(\mathbb{C}^{\ell+1}, \beta, \underline{\lambda}\right) .
$$




\section{2. $S^{1} \times U_{\ell+1}$-equivariant volume of $\mathcal{M}\left(D, \mathbb{C}^{\ell+1}\right)$}

In this subsection, we provide an interpretation of the functional integral calculation in Section 2 as a calculation of an equivariant volume of a space of holomorphic maps of the disk $D$ into $\mathbb{C}^{\ell+1}$.

Let $\mathcal{M}\left(D, \mathbb{C}^{\ell+1}\right)$ be the space of holomorphic map of $D=\{z|| z \mid \leq 1\}$ into $\mathbb{C}^{\ell+1}$. An element of $\mathcal{M}\left(D, \mathbb{C}^{\ell+1}\right)$ can be described by a set of functions $\left(\varphi^{j}(z, \bar{z}), \bar{\varphi}^{j}(z, \bar{z})\right)$, satisfying the constraints

$$
\partial_{\bar{z}} \varphi^{j}(z, \bar{z})=0, \quad \partial_{z} \bar{\varphi}^{j}(z, \bar{z})=0, \quad j=1, \ldots,(\ell+1)
$$

Define a symplectic form on the space $\mathcal{M}\left(D, \mathbb{C}^{\ell+1}\right)$ of holomorphic maps as follows:

$$
\Omega=\frac{\imath}{2} \sum_{j=1}^{\ell+1} \int_{0}^{2 \pi} \delta \varphi^{j}(\sigma) \wedge \delta \bar{\varphi}^{j}(\sigma) d \sigma
$$

where $\varphi^{j}(\sigma), \bar{\varphi}^{j}(\sigma)$ are restrictions of $\varphi^{j}(z, \bar{z}), \bar{\varphi}^{j}(z, \bar{z})$ to the boundary $\partial D=S^{1}$. This symplectic form is invariant with respect to the group $S^{1}$ of loop rotations $\sigma \rightarrow \sigma+\alpha$ and the action of $U_{\ell+1}$ is induced from the standard action on $\mathbb{C}^{\ell+1}$. The action of $S^{1} \times U_{\ell+1}$ is Hamiltonian and the momenta corresponding to the action of $S^{1}$ and of the Cartan subgroup $U_{1}^{\ell+1} \subset U_{\ell+1}$ are given by

$$
\begin{aligned}
H_{0} & =\frac{\imath}{2} \sum_{j=1}^{\ell+1} \int_{0}^{2 \pi} \bar{\varphi}^{j}(\sigma) \partial_{\sigma} \varphi^{j}(\sigma) d \sigma, \quad H_{j}=-\frac{1}{2} \int_{0}^{2 \pi}\left|\varphi^{j}(\sigma)\right|^{2} d \sigma \\
j & =1, \ldots, \ell+1
\end{aligned}
$$

In the infinite-dimensional setting we can try to define an analog of the equivariant volume (3.2). We use an analog of the representation (3.9) in terms of the integral over commuting and anticommuting variables. Let $\chi^{j}(z, \bar{z})$ and $\bar{\chi}^{j}(z, \bar{z})$ be anticommuting counterparts of $\varphi^{j}(z, \bar{z}) \bar{\varphi}^{j}(z, \bar{z})$ satisfying the equations

$$
\partial_{\bar{z}} \chi^{j}(z, \bar{z})=0, \quad \partial_{z} \bar{\chi}^{j}(z, \bar{z})=0, \quad j=1, \ldots,(\ell+1)
$$

Let $\hbar$ be a generator of Lie algebra of $S^{1}$ and $\underline{\lambda}=\left(\lambda_{1}, \ldots, \lambda_{\ell+1}\right)$ be generators of Cartan subalgebra of $\mathfrak{u}_{\ell+1}$. The following formal integral should be considered as a $S^{1} \times U_{\ell+1}$-equivariant volume of the space of holomorphic 
$\operatorname{maps} \mathcal{M}\left(D, \mathbb{C}^{\ell+1}\right)$

$$
\begin{aligned}
& Z\left(\mathcal{M}\left(D, \mu, \mathbb{C}^{\ell+1}\right), \beta, \underline{\lambda}\right) \\
& \quad=\int_{\Pi T \mathcal{M}\left(D, \mathbb{C}^{\ell+1}\right)} d m(\varphi, \chi) \mathrm{e}^{\frac{\imath \mu}{2} \sum_{j=1}^{\ell+1}\left(\int_{0}^{2 \pi} d \sigma \chi^{j} \bar{\chi}^{j}+\imath \lambda_{j} H_{j}\right)+\hbar H_{0}},
\end{aligned}
$$

where $H_{0}, H_{j}$ are given by $(3.17)$ and $d m(\varphi, \chi)$ is an integration measure to be defined. This functional integral is a product of functional integrals (2.47), (2.48) arising at an intermediate step of the proof of Proposition 2.1. Thus we can define the measure $d m(\varphi, \chi)$ in (3.19) in the same way as in Proposition 2.1. Finally, taking into account Theorem 2.1 we arrive to the conclusion that the r.h.s. of the relation (2.25) is indeed can be considered as $S^{1} \times U_{\ell+1}$-equivariant volume of the space of holomorphic maps $\mathcal{M}\left(D, \mathbb{C}^{\ell+1}\right)$. Thus taking into account the definition of the equivariant symplectic volume given above, Proposition 2.1 can be reformulated as follows. The classical $\Gamma$-function is equal to the $S^{1} \times U(1)$-equivariant symplectic volume of the space $\mathcal{M}(D, \mathbb{C})$ of holomorphic maps of the disk $D$ to the complex plane $\mathbb{C}$.

Let us push further the analogy with the finite-dimensional case considered in the previous subsection. We would like to find a quantum system such that the equivariant volume (3.19) appears in the assymptotic of its partition function. This is easy to accomplish taking into account that underlying infinite-dimensional symplectic space $\mathcal{M}\left(D, \mathbb{C}^{\ell+1}\right)$ is a linear space. Consider the Fourier series expansion

$$
\varphi^{j}(\sigma)=\sum_{n \geq 0}(2 \pi)^{-1 / 2} \varphi_{n}^{j} e^{\imath n \sigma}, \quad \bar{\varphi}^{j}(\sigma)=\sum_{n \geq 0}(2 \pi)^{-1 / 2} \bar{\varphi}_{n}^{j} \mathrm{e}^{-\imath n \sigma}
$$

In terms of the Fourier coefficients we have

$$
\begin{gathered}
\Omega=\frac{\imath}{2} \sum_{j=1}^{\ell+1} \sum_{n \geq 0} \delta \varphi_{n}^{j} \wedge \delta \bar{\varphi}_{n}^{j} \\
H_{0}=-\frac{1}{2} \sum_{j=1}^{\ell+1} \sum_{n>0}^{\infty} n\left|\varphi_{n}^{j}\right|^{2}, \quad H_{j}=-\frac{1}{2} \sum_{n \geq 0}^{\infty}\left|\varphi_{n}^{j}\right|^{2} .
\end{gathered}
$$

Quantization of the algebra of polynomial functions on $\mathcal{M}\left(D, \mathbb{C}^{\ell+1}\right)$ with the symplectic structure (3.21) is given by

$$
\left[\varphi_{n}^{j}, \varphi_{m}^{k}\right]=0, \quad\left[\bar{\varphi}_{n}^{j}, \varphi_{m}^{k}\right]=-2 \tilde{\hbar} \delta^{j k} \delta_{n m}, \quad\left[\bar{\varphi}_{n}^{j}, \bar{\varphi}_{m}^{k}\right]=0 .
$$


We chose a polarization in such a way that the Hilbert space $\mathcal{H}^{(\ell+1)}$ is realized as a space of functions of $\varphi_{n}^{j}, n \geq 0, j=1, \ldots,(\ell+1)$. Then $\bar{\varphi}_{n}^{j}$, $n \geq 0, j=1, \ldots,(\ell+1)$ act as first-order differential operators

$$
\bar{\varphi}_{n}^{j}=-2 \tilde{\hbar} \frac{\partial}{\partial \varphi_{n}^{j}} .
$$

The Hilbert space $\mathcal{H}^{(\ell+1)}$ has a natural action of $S^{1} \times\left(U_{1}\right)^{\ell+1}$ induced by the action on the linear coordinates

$$
\begin{aligned}
& \mathrm{e}^{\imath \alpha_{0}}: \varphi_{n}^{j} \rightarrow \mathrm{e}^{\imath n \alpha_{0}} \varphi_{n}^{j}, \quad \mathrm{e}^{\imath \alpha_{0}} \in S^{1}, \\
& \mathrm{e}^{\imath \alpha_{k}}: \varphi_{n}^{j} \rightarrow \mathrm{e}^{\imath \delta_{k j} \alpha_{j}} \varphi_{n}^{j}, \quad \mathrm{e}^{\imath \alpha_{k}} \in U_{1} .
\end{aligned}
$$

The action of the corresponding Lie algebra is generated by the vector fields

$$
\hat{H}_{0}=\tilde{\hbar} \sum_{j=1}^{\ell+1} \sum_{n=0}^{\infty} n \varphi_{n}^{j} \frac{\partial}{\partial \varphi_{n}^{j}}, \quad \hat{H}_{j}=\tilde{\hbar} \sum_{n=0}^{\infty} \varphi_{n}^{j} \frac{\partial}{\partial \varphi_{n}^{j}} .
$$

Thus the infinite-dimensional counterpart of (3.13) is given by the following partition function:

$$
Z_{\tilde{\hbar}}\left(\mathcal{M}\left(D, \mathbb{C}^{\ell+1}, \beta, \underline{\lambda}\right)=\operatorname{Tr}_{\mathcal{H}^{(\ell+1)}} \mathrm{e}^{-\frac{\beta}{\hbar}\left(\hbar \hat{H}_{0}+\sum_{j=1}^{\ell+1} \lambda_{j} \hat{H}_{j}\right)}\right.
$$

where $\underline{\lambda}=\left(\lambda_{1}, \ldots, \lambda_{\ell+1}\right)$.

Proposition 3.1. The following identity holds

$$
Z_{\tilde{\hbar}}\left(\mathcal{M}\left(D, \mathbb{C}^{\ell+1}, \beta, \underline{\lambda}\right)=\prod_{j=1}^{\ell+1} \Gamma_{q}\left(t_{j}\right)\right.
$$

where $q=\mathrm{e}^{-\beta \hbar}, t_{j}=\mathrm{e}^{-\beta \lambda_{j}}$ and the $q$-version of $\Gamma$-function is given by

$$
\Gamma_{q}(t)=\prod_{k=0}^{+\infty} \frac{1}{1-t q^{k}}
$$

Proof. Direct calculation.

Note that the first order differential operators (3.25) can be considered as a quantization of the Hamiltonians (3.22). Moreover, similarly to (3.14) the asymptotic of $(3.26)$ is proportional to the equivariant symplectic volume 
(3.19). Thus the proposed $q$-version of $\Gamma$-function is obtained by applying the standard quantization rules to the classical $\Gamma$-function expressed as an equivariant volume integral.

Remark 3.1. The Hilbert space $\mathcal{H}^{(\ell+1)}$ can be considered as a Hilbert space of a second quantized system, i.e., $\mathcal{H}^{(\ell+1)}=S^{*} V$ is a space of multiparticle states where $V$ is a one-particle Hilbert space. Explicitly $\mathcal{H}^{(\ell+1)}=$ $\mathbb{C}\left[\varphi_{n}^{j}\right], n \geq 0, j=1, \ldots,(\ell+1)$ is a space of polynomial functions on $\mathbb{C}^{\ell+1} \otimes$ $\mathbb{C}[\xi]$ and $V \subset \mathcal{H}^{(\ell+1)}$ is a subspace of linear functions on $\mathbb{C}^{\ell+1} \otimes \mathbb{C}[\xi]$.

\section{4. $q$-deformed $\Gamma$-function: three-dimensional interpretation}

In the previous sections, we propose the integral representation of the $\Gamma$-function as a functional integral in the equivariant topological two-dimensional linear sigma model. This integral representation can be interpreted as an equivariant symplectic volume of the space of holomorphic maps of a disk into a complex plain $\mathbb{C}$. We also define a natural quantum version of the equivariant symplectic volume. This is equal to a $q$-deformed $\Gamma$-function expressed as a partition function of a quantum system. It is natural to look for a topological field theory expression for $q$-deformed $\Gamma$-function similar to the expression for classical $\Gamma$-function given in Section 2. In this Section we propose such a formulation in terms of an equivariant topological threedimensional linear sigma model. This is an example of the representation of a $K$-theory analog of GW invariants $[29,31]$ in terms of three-dimensional topological field theories discussed below.

\subsection{Path integral representation for the character}

We start with a path integral representation of the partition function (3.13) of the quantum system obtained by a quantization of the classical phase space $\mathbb{C}^{\ell+1}$ supplied with the symplectic form (3.3). We have the following standard representation:

$$
Z_{\tilde{\hbar}}\left(\mathbb{C}^{\ell+1}, \beta, \underline{\lambda}\right)=\int_{L \Pi T\left(\mathbb{C}^{\ell+1}\right)}[D z][D \bar{z}][D \eta][D \bar{\eta}] \mathrm{e}^{S_{Q M}(z, \bar{z}, \eta, \bar{\eta})},
$$

where

$$
S_{Q M}=\frac{1}{2 \tilde{\hbar}} \sum_{j=1}^{\ell+1} \int_{0}^{2 \pi} d \tau\left(\bar{z}^{j} \frac{d z^{j}}{d \tau}+\frac{\imath \beta}{2 \pi}\left(\eta_{j} \bar{\eta}_{j}+\imath \lambda_{j}\left|z^{j}\right|^{2}\right)\right)
$$


Here commuting periodic functions $\left(z^{j}(\tau), \bar{z}^{j}(\tau)\right), j=1, \ldots,(\ell+1)$ and anticommuting periodic functions $\left(\eta^{j}(\tau), \bar{\eta}^{j}(\tau)\right), j=1, \ldots,(\ell+1)$ parametrize the loop space of the odd tangent bundle $\Pi T \mathbb{C}^{\ell+1}=\mathbb{C}^{\ell+1 \mid \ell+1}$ to $\mathbb{C}^{\ell+1}$. The role of the integral over anticommuting variables is to provide a proper integration measure over commuting variables (see the appendix).

The path integral (4.1) is a Gaussian one. By the definition we use, it is expressed through the determinants of the differential operators regularized using the corresponding $\zeta$-functions. The integral (4.1) is easily calculated using the expression (A.10) for regularized determinants

$$
Z_{\tilde{\hbar}}\left(\mathbb{C}^{\ell+1}, \underline{\lambda}\right)=\prod_{j=1}^{\ell+1} \frac{1}{\operatorname{det}\left(\frac{d}{d \tau}-\beta \lambda_{j} / 2 \pi\right)}=\prod_{j=1}^{\ell+1} \frac{1}{1-\mathrm{e}^{-\beta \lambda_{j}}} .
$$

This coincides with the result of the direct calculation of the partition function (3.13). Let us remark that the $\zeta$-function regularization implies the following normal ordering: given a monomial in $z^{j}, \bar{z}^{j}, j=1, \ldots, \ell+1$, one should put $\bar{z}^{j}$ 's to the l.h.s. and $z^{j}$ 's to the r.h.s.

The functional integral (4.1) can be related with equivariant cohomology of the loop space $L \mathbb{C}^{\ell+1}$ (see e.g. [11]). Let us identify differential forms on $L \mathbb{C}^{\ell+1}$ with functions on the loop space of $\mathbb{C}^{\ell+1 \mid \ell+1}$. Let $\left(z^{j}(\tau), \bar{z}^{j}(\tau)\right), j=$ $1, \ldots,(\ell+1)$ and anticommuting functions $\left(\eta^{j}(\tau), \bar{\eta}^{j}(\tau)\right), j=1, \ldots,(\ell+1)$ be linear coordinates on $L\left(\Pi T \mathbb{C}^{\ell+1}\right)$. The standard de Rham differential on $L \mathbb{C}^{\ell+1}$ is then given by

$$
\delta z^{j}(\tau)=\eta^{j}(\tau), \quad \delta \eta^{j}(\tau)=0, \quad \delta \bar{z}^{j}(\tau)=\bar{\eta}^{j}(\tau), \quad \delta \bar{\eta}^{j}(\tau)=0
$$

We have a natural diagonal action of $\left(U_{1}\right)^{\ell+1}$ on $L\left(\Pi T \mathbb{C}^{\ell+1}\right)$ induced by the diagonal action on $\mathbb{C}^{\ell+1}$. Let $S_{\tau}^{1}$ act by rotations of the loops $\tau \rightarrow \tau+$ $\alpha, \alpha \in[0,2 \pi]$ and let $\tilde{\beta}$ be a generator of the corresponding Lie algebra. Let $G_{0}=S_{\tau}^{1} \times\left(U_{1}\right)^{\ell+1}$ be an abelian subgroup of $G=S_{\tau}^{1} \times U_{\ell+1}$. Then $G_{0^{-}}$ equivariant de Rham differential is given by

$$
\begin{aligned}
& \delta_{G_{0}} z^{j}(\tau)=\eta^{j}(\tau), \quad \delta_{G_{0}} \eta^{j}(\tau)=-\left(\tilde{\beta}^{-1} \frac{d z^{j}}{d \tau}+\imath \lambda_{j} z^{j}\right), \\
& \delta_{G_{0}} \bar{z}^{j}(\tau)=\bar{\eta}^{j}(\tau), \quad \delta_{G_{0}} \bar{\eta}^{j}(\tau)=-\left(\tilde{\beta}^{-1} \frac{d \bar{z}^{j}}{d \tau}-\imath \lambda_{j} \bar{z}^{j}\right),
\end{aligned}
$$

and satisfy the relation

$$
\delta_{G_{0}}^{2}=-\tilde{\beta}^{-1} \partial_{\tau}
$$


The action functional $(4.2)$ is proportional to the $G_{0}$-equivariant extension $\omega_{G}$ of the symplectic form $(3.8)$

$$
\omega_{G_{0}}=\frac{\imath}{2} \sum_{j=1}^{\ell+1} \int_{0}^{2 \pi} d \tau\left(\bar{\eta}^{j} \eta^{j}+\imath \lambda_{j}\left|z^{j}\right|^{2}+\tilde{\beta}^{-1} \bar{z}^{j} \frac{d z^{j}}{d \tau}\right),
$$

where $\tilde{\beta}=\imath \beta / 2 \pi$. Thus the functional integral with the action (4.2) can be considered as (an analytic continuation of) a $G_{0}$-equivariant symplectic volume of the loop space $L \mathbb{C}^{\ell+1}$.

\subsection{Three-dimensional interpretation of $K$-theory GW invariants}

Givental in [29] (see also [31]) proposed a construction of quantum $K$ theory invariants of complex manifolds generalizing quantum cohomology invariants arising in GW theory. The Givental construction of quantum $K$-theory invariants of a manifold $X$ is formulated directly in terms of the characteristic classes of complexes of coherent sheaves on compactified moduli space of holomorphic curves in $X$. For example let $X$ be a Fano manifold with the cohomology ring generated by the second cohomology $H^{2}(X), \operatorname{dim} H^{2}(X)=m$. Let $\mathcal{M}_{\underline{d}}(\Sigma, X)$ be a compactified moduli space of holomorphic maps of an algebraic curve $\Sigma$ into $X$ of a fixed multi-degree $\underline{d}=\left(d_{1}, \ldots, d_{m}\right) \in \mathbb{Z}^{m}$. The corresponding invariant is a generating function of holomorphic Euler characteristics of line bundles on $\mathcal{M}_{\underline{d}}(\Sigma, X)$

$$
\mathcal{G}(X, \underline{Q}, \mathcal{L})=\sum_{\underline{d} \in \mathbb{Z}^{m}} \operatorname{ch}\left(\mathcal{M}_{\underline{d}}(\Sigma, X), \mathcal{L}\right) \prod_{a=1}^{m} Q_{a}^{d_{a}}
$$

where

$$
\operatorname{ch}\left(\mathcal{M}_{\underline{d}}(\Sigma, X), \mathcal{L}\right)=\sum_{n}(-1)^{n} \operatorname{dim} H^{n}\left(\mathcal{M}_{\underline{d}}(\Sigma, X), \mathcal{L}\right),
$$

and $\mathcal{L}$ is a line bundle on $\mathcal{M}_{\underline{d}}(\Sigma, X)$.

In this subsection, we propose a topological field theory interpretation of the quantum $K$-theory invariants. Les us start with some heuristic arguments. Cohomology of a manifold $X$ can be described in terms of differential forms on $X$ via the de Rham complex. On the other hand $K$-cohomology of $X$ can be described in terms of vectors bundles on $X$. Thus a relation between cohomology and $K$-theory can be considered as a kind of a quantization. The general approach to quantization is via Feynman path integral. 
The Feynman path integral description of quantum mechanics implies that a quantization of a symplectic manifold should be described in terms of the geometry of the loop space on the symplectic manifold. Therefore $K$-theory of $X$ (modulo torsion) should allow interpretation in terms of cohomology of the loop space $L X$. Let us apply this reasoning to quantum counterpart of cohomology and $K$-theory. The quantum cohomology of a manifold $X$ are described in terms of a topological sigma model in two dimensions with the target space $X[46]$. Thus it would be natural to guess that quantum $K$-theory invariants should be described in terms of two-dimensional sigma model on the loop space $L X$, i.e., in terms of three-dimensional topological sigma models. We propose such three-dimensional topological theory description below.

Let $\Sigma$ be a Riemann surface and $X$ be a Kähler manifold. We retain the basic notations introduced in Section 2.1. Let us consider a three-dimensional manifold $\Sigma \times S^{1}$. We pick a metric $d^{2} s_{\Sigma}=h_{z \bar{z}} d z d \bar{z}$ on $\Sigma$ and a constant metric $d^{2} s_{S^{1}}=d \tau^{2}$ on $S^{1}$. Let $\pi: \Sigma \times S^{1} \rightarrow \Sigma$ be a projection. Introduce the fields $F$ and $\bar{F}$ as sections of $\pi^{*}(K) \otimes \Phi^{*}\left(T^{0,1}\right)$ and of $\pi^{*}(\bar{K}) \otimes \Phi^{*}\left(T^{1,0}\right)$ correspondingly. The anticommuting fields $\chi, \bar{\chi}$ are sections of the bundles $\Phi^{*}\left(\Pi T^{1,0} X\right), \Phi^{*}\left(\Pi T^{0,1}\right)$ and anticommuting fields $\psi, \bar{\psi}$ are sections of the bundles $\pi^{*}(K) \otimes \Phi^{*}\left(\Pi T^{0,1}\right), \pi^{*}(\bar{K}) \otimes \Phi^{*}\left(\Pi T^{1,0}\right)$ respectively. Metrics $g$ on $X$ and $h$ on $\Sigma$ induce a Hermitian paring $\langle$,$\rangle on the space of sections of the$ considered bundles. An example of the pairing in local coordinates is given by (2.2). Consider the following action functional in three dimensions:

$$
\begin{aligned}
S_{0}^{3 d}(t)= & \beta \int_{S^{1} \times \Sigma} d \tau d^{2} z \sqrt{h}(\imath\langle\mathcal{F}, \bar{\partial} \varphi\rangle+\imath\langle\overline{\mathcal{F}}, \partial \bar{\varphi}\rangle+\imath\langle\bar{\psi}, D \bar{\chi}\rangle+\imath\langle\psi, \bar{D} \chi\rangle \\
& +t\langle\mathcal{F} \mathcal{F}\rangle+\frac{t}{2}\left\langle\psi, \partial_{\tau} \psi\right\rangle+\frac{t}{2}\left\langle\psi, \partial_{\tau} \psi\right\rangle+t\langle\bar{\psi}, R(\psi, \bar{\chi}) \chi\rangle \\
& \left.+2 \tilde{t} \beta^{-2}\left\langle\partial_{\tau} \varphi, \partial_{\tau} \varphi\right\rangle+\imath \tilde{t} \beta^{-1}\left\langle\chi, \partial_{\tau} \chi\right\rangle+\imath \tilde{t} \beta^{-1}\left\langle\bar{\chi}, \partial_{\tau} \bar{\chi}\right\rangle\right) .
\end{aligned}
$$

The fields $\mathcal{F}, \overline{\mathcal{F}}$ and the derivatives $D_{z}, \bar{D}_{\bar{z}}$ are locally defined as in (2.5), (2.6). The action functional (4.8) is invariant with respect to the transformations

$$
\begin{array}{llll}
\delta \varphi=\chi, & \delta \chi=-\imath \beta^{-1} \partial_{\tau} \varphi, & \delta \bar{\psi}=\bar{F}, & \delta \bar{F}=-\imath \beta^{-1} \partial_{\tau} \bar{\psi} \\
\delta \bar{\varphi}=\bar{\chi}, & \delta \bar{\chi}=-\imath \beta^{-1} \partial_{\tau} \bar{\varphi}, & \delta \psi=F, & \delta F=-\imath \beta^{-1} \partial_{\tau} \psi
\end{array}
$$

and the following relation holds

$$
\delta^{2}=-\imath \beta^{-1} \partial_{\tau}
$$


Similarly to the action $S_{0}(t)$ in (2.3) the three-dimensional action (4.8) can be written as follows

$$
S_{0}^{3 d}(t)=\beta \int_{S^{1} \times \Sigma} d \tau d^{2} z \sqrt{h} \delta \mathcal{V}(t),
$$

where

$$
\mathcal{V}(t)=\left\langle\psi, \frac{1}{2} t \mathcal{F}+\imath \bar{\partial} \varphi\right\rangle+\imath \tilde{t} \beta^{-1}\left\langle\chi, \partial_{\tau} \varphi\right\rangle+\left\langle\bar{\psi}, \frac{1}{2} t \overline{\mathcal{F}}+\imath \partial \bar{\varphi}\right\rangle+\imath \tilde{t} \beta^{-1}\left\langle\bar{\chi}, \partial_{\tau} \bar{\varphi}\right\rangle
$$

Note that the form of the $\delta$-transformations allows to interpret the threedimensional sigma model (4.8) in terms of $S^{1}$-equivariant cohomology where $S^{1}$ acts by shits of $\tau$.

Remark 4.1. The action (4.8) can be obtained by a twisting of a threedimensional $\mathcal{N}=2$ SUSY sigma model on $\Sigma \times S^{1}$ with the target space $X$ similarly to the two-dimensional case [46].

We consider the theory with the action (4.8) deformed by a $\delta$-closed functional. Let $\mathcal{L}$ be a holomorphic line bundle on $X$ with a curvature twoform $\omega^{\mathcal{L}}$ representing the first Chern class $c_{1}(\mathcal{L})$. Locally we can express the curvature in terms of a connection one-form $\alpha$

$$
\omega^{\mathcal{L}}=\bar{\partial} \alpha
$$

The following functionals are invariant with respect to transformations (4.9):

$$
\begin{aligned}
& \exp (2 \pi \imath \mathcal{O}(\mathcal{L})) \\
& =\exp \left(2 \pi \imath \int_{\Sigma \times S^{1}} d \tau \sqrt{h} d^{2} z\left(\sum_{i, \bar{j}=1}^{\ell+1} \omega_{i \bar{j}}^{\mathcal{L}} \chi^{i} \chi^{\bar{j}}+\sum_{i=1}^{\ell+1} \alpha_{i}(\varphi) \partial_{\tau} \varphi^{i}\right)\right) \\
& \quad \exp \left(\mathcal{O}_{\omega}(y)\right)=\exp \left(\sum_{a=1}^{\operatorname{rk} H^{2}(X)} y_{a} \int_{0}^{2 \pi} d \tau \int_{\Sigma} \Phi^{*}\left(\omega_{a}\right)\right) .
\end{aligned}
$$

where $\left\{\omega_{a}\right\}$ is a bases in $H^{2}(X)$. Note that the observable (4.13) is well defined. Indeed, although it is written using the local representation (4.12) the ambiguity is given by the exponent of the $2 \pi \imath$ multiplied by a period of the two-form $\omega^{\mathcal{L}} \in H^{2}(X, \mathbb{Z})$ and thus irrelevant. 
The three-dimensional sigma model with the action (4.8) is not well defined in general as a quantum field theory. The sigma model provides a long wave length ("infra-red") description and needs a proper short wave length ("ultraviolent") completion in general. For the case of the target space being a flag manifold (relevant to the quantum $K$-theory invariants considered in [31]) the proper completion can be given for example in terms of quiver gauge theories with $\mathcal{N}=4$ SUSY. We leave the detailed construction of these completions to another occasion. Here, we provide heuristic arguments to support the conjecture that the topological field theory with the action (4.8) specialized to $t=0$ calculates quantum $K$-theory invariants (4.7). The argument is very close to that for the standard description of GW invariants in terms of a two-dimensional twisted $\mathcal{N}=2$ SUSY sigma model $[4,46]$. Integrating over $F$ and $\psi$ we reduce the integration over other fields to the vicinity of the subspace of maps $\Sigma \times S^{1} \rightarrow X$ holomorphic along $\Sigma$

$$
\partial_{\bar{z}} \varphi^{i}=0, \quad D_{\bar{z}} \chi^{i}=0
$$

The corresponding determinant contributions arising from the integration over commuting and anticommuting fields in the quadratic approximation around the subspace (4.15) cancel each other (we consider the situation when there is no zero modes of $F$ and $\psi$ ). Thus the integral reduces to the path integral with the following action:

$$
\begin{aligned}
S_{\mathrm{red}}(\mathcal{L})= & \int_{0}^{2 \pi} d \tau\left[\int _ { \Sigma } \sqrt { h } d ^ { 2 } z \left(\sum_{i, \bar{j}=1}^{\ell+1} \tilde{t} \omega_{i \bar{j}}^{\mathcal{L}}\left(\beta^{-2} \frac{\partial \varphi^{i}}{\partial \tau} \frac{\partial \bar{\varphi}^{\bar{j}}}{\partial \tau}+\beta^{-1} \bar{\chi}^{\bar{j}} \frac{\partial \chi^{i}}{\partial \tau}\right)\right.\right. \\
& \left.\left.+\left(\sum_{i, \bar{j}=1}^{\ell+1} \omega_{i \bar{j}}^{\mathcal{L}} \chi^{i} \bar{\chi}^{\bar{j}}+\sum_{i=1}^{\ell+1} \alpha_{i}(\varphi) \partial_{\tau} \varphi^{i}\right)\right)+\sum_{a=1}^{\operatorname{rk} H^{2}(X)} y_{a} \int_{\Sigma} \Phi^{*}\left(\omega_{a}\right)\right]
\end{aligned}
$$

where $\varphi: \Sigma \rightarrow X$ is a holomorphic map and $\chi$ is a section of the odd tangent bundle $\Pi \operatorname{TM}(\Sigma, X)$ to the space $\mathcal{M}(\Sigma, X)$ of the holomorphic maps. The theory described by this action is a $\mathcal{N}=1 / 2$ SUSY one-dimensional sigma model with the target space $\mathcal{M}(\Sigma, X)$ (first line in (4.16)) deformed by an observable (second line in (4.16)). Using the standard facts on the partition functions for $\mathcal{N}=1 / 2$ SUSY quantum mechanics (see $[2,3,8,17]$ ) we can identify the result of taking functional integral with the action (4.16) with the generating function (4.7) given by the sum of holomorphic Euler characteristics of the line bundle $\mathcal{L}$ with $\omega=c_{1}(\mathcal{L})$ over components $\mathcal{M}_{\underline{d}}(\Sigma, X)$ of 
the moduli space of holomorphic maps with the identification $Q_{j}=\exp y_{j}$. It is easy to generalize these considerations to the case of an equivariant three-dimensional sigma model taking into account proper ultraviolent completion. In the next subsection, we consider an example of an equivariant three-dimensional topological linear sigma model which is a well-defined quantum field theory by itself.

\subsection{Equivariant linear sigma model on $D \times S^{1}$}

In this subsection, we consider an equivariant version of the topological linear sigma model on a non-compact three-dimensional space $D \times S^{1}$ with the target space $X=\mathbb{C}^{\ell+1}$. We pick the flat metric $(2.17)$ on the disk and the symplectic structure $(3.3)$ on $\mathbb{C}^{\ell+1}$. Let $U_{\ell+1}$ act on $\mathbb{C}^{\ell+1}$ via the standard representation and $S^{1}$ act on the first factor in $D \times S^{1}$ by rotations as it was introduced in Section 2. We would like to consider $S^{1} \times$ $U_{\ell+1}$-equivariant version of the three-dimensional topological field theory introduced in Section 4.2. Following the reasoning of the previous section we consider an equivariance with respect to a Cartan subgroup $G_{0}=S^{1} \times$ $\left(U_{1}\right)^{\ell+1}$ of $G=S^{1} \times U_{\ell+1}$. The $G_{0}$-equivariant modification of the transformations (4.9) is given by

$$
\begin{array}{ll}
\delta_{G_{0}} \varphi=\chi, & \delta_{G_{0}} \chi=-\left(\hbar \partial_{\sigma}+2 \pi \imath \beta^{-1} \partial_{\tau}+\imath \lambda\right) \varphi, \\
\delta_{G_{0}} \psi_{\bar{z}}=F_{\bar{z}}, & \delta_{G_{0}} F_{\bar{z}}=-\left(\hbar \partial_{\sigma}+2 \pi \imath \beta^{-1} \partial_{\tau}+\imath \lambda\right) \psi_{\bar{z}} .
\end{array}
$$

Consider the $\delta_{G_{0}}$-invariant action (4.8) with $\tilde{t}=t=0$ on $N=S^{1} \times D$ specialized to the case of $X=\mathbb{C}^{\ell+1}$

$$
\begin{aligned}
S_{0} & =\int_{S^{1} \times D} d^{2} z d \tau \delta_{G_{0}} \mathcal{V} \\
& =\imath \int_{S^{1} \times D} d^{2} z d \tau\left(\partial_{\bar{z}} \chi \bar{\psi}_{z}+\bar{F}_{z} \partial_{\bar{z}} \varphi+\partial_{z} \bar{\chi} \psi_{\bar{z}}+F_{\bar{z}} \partial_{z} \bar{\varphi}\right)
\end{aligned}
$$

where

$$
\mathcal{V}=\partial_{\bar{z}} \varphi \bar{\psi}_{z}+\partial_{z} \bar{\varphi} \psi_{\bar{z}}
$$

We deform the action by a $\delta_{G_{0}}$ - and $G_{0}$-invariant observable on the boundary $\partial N=S^{1} \times S^{1}$

$$
S=S_{0}+\mathcal{O}
$$

where

$$
\mathcal{O}=\frac{\imath}{2} \beta \int_{\partial N=S^{1} \times S^{1}} d \tau d \sigma\left(\bar{\chi} \chi+\bar{\varphi}\left(\hbar \partial_{\sigma}+2 \pi \imath \beta^{-1} \partial_{\tau}+\imath \lambda\right) \varphi\right)
$$


The integration over $F$ and $\psi$ localizes the functional integral to the subspace of maps $N \rightarrow \mathbb{C}^{\ell+1}$ satisfying the equations

$$
\partial_{\bar{z}} \varphi=0, \quad \partial_{z} \bar{\varphi}=0, \quad \partial_{\bar{z}} \chi=0, \quad \partial_{z} \bar{\chi}=0 .
$$

Let us use the expansion of the solutions of the constraints (4.20)

$$
\begin{array}{ll}
\varphi(z, \bar{z}, \tau)=\sum_{n=0}^{+\infty}(2 \pi)^{-1 / 2} \varphi_{n}(\tau) z^{n}, & \bar{\varphi}(z, \bar{z}, \tau)=\sum_{n=0}^{+\infty}(2 \pi)^{-1 / 2} \varphi_{n}(\tau) \bar{z}^{n}, \\
\chi(z, \bar{z}, \tau)=\sum_{n=0}^{+\infty}(2 \pi)^{-1 / 2} \chi_{n}(\tau) z^{n}, & \bar{\chi}(z, \bar{z}, \tau)=\sum_{n=0}^{+\infty}(2 \pi)^{-1 / 2} \chi_{n}(\tau) \bar{z}^{n} .
\end{array}
$$

Then the integral factorizes into the product of elementary integrals

$$
Z=\prod_{n=0}^{\infty} Z_{n}
$$

where

$$
Z_{n}=\int\left[D \varphi_{n}\right]\left[D \bar{\varphi}_{n}\right]\left[D \chi_{n}\right]\left[D \bar{\chi}_{n}\right] \mathrm{e}^{-S_{n}}
$$

and

$$
S_{n}=-\frac{\imath}{2} \beta \int_{0}^{2 \pi} d \tau\left(\bar{\chi}_{n} \chi_{n}+\bar{\varphi}_{n}\left(2 \pi \imath \beta^{-1} \partial_{\tau}+\imath \hbar n+\imath \lambda\right) \varphi_{n}\right) .
$$

Each $Z_{n}$ is a path integral of the form (4.1) and thus using previous calculations we have

$$
Z_{n}=\frac{1}{1-\mathrm{e}^{-\beta(\hbar n+\lambda)}}
$$

Finally for the partition function of the three-dimensional topological linear sigma model on $S^{1} \times D$, we obtain

$$
Z=\prod_{n=0}^{+\infty} \frac{1}{1-t q^{n}}
$$

where $t=\mathrm{e}^{-\beta \lambda}, q=\mathrm{e}^{-\beta \hbar}$. This coincides with the representation given in Proposition 3.1.

\section{Conclusion}

To conclude this paper we outline some directions for future research. 
In this paper, we consider equivariant topological sigma models on a disk such that the equivariance group includes the group $S^{1}$ of disk rotations. It is natural to expect that this $S^{1}$-equivariance is a remnant of $\operatorname{Diff}\left(S^{1}\right)$ equivariance of two-dimensional topological quantum gravity. The relation between $S^{1}$-equivariance and topological gravity is well known. For example in $[27,28]$, the correlation functions of $S^{1}$-equivariant topological sigma models on $\mathbb{P}^{1}$ are expressed through the correlation functions of the topological sigma model coupled with the topological quantum gravity. Thus one should expect that the results of this papers can be put in the framework of a first quantized topological string theory. However, let us note that there are also indications that the proper interpretation of our results should be in terms of a second quantized topological string field theory. The simplest soluble example of the topological string theory is given by a pure topological gravity completely solved in [34]. This solution can be reformulated in terms of a quantum field theory on a disk with a quadratic action playing the role of the second quantized string theory $[19,26]$. In particular, such a formulation provides an intriguing analogy with the considerations of this note and seems to deserve further considerations.

The construction of the functional integral representation of local Archimedean $L$-factors uses an integral representation of a $\Gamma$-function (see Proposition 2.1). Thus classical $\Gamma$-function is equal to equivariant volume of the space of holomorphic maps of the disk to complex plain. This functional integral representation should be compared with the standard Euler integral representation. As we demonstrate in [24] the Euler integral representation naturally arises as a disk partition function in the equivariant type $B$ topological Landau-Ginzburg model on a disk with the target space $\mathbb{C}$ and the superpotential $W(\xi)=e^{\xi}+\lambda \xi, \xi \in \mathbb{C}$. This result is not surprising in view of the mirror symmetry between the type $\mathrm{A}$ and type $\mathrm{B}$ topological sigma model (see [33] for detailed discussion). Thus we have two integral representations of $\Gamma$-function in terms of an infinite-dimensional equivariant symplectic volume and in terms of an finite-dimensional complex integral. Taking into account the mirror symmetry relating the two underlying topological theories, these two integral representations should be considered on equal footing.

These two different integral representations of $\Gamma$-functions are similar to two constructions of the local Archimedean $L$-factors discussed in Section 1. The equivalence of the resulting $L$-factors is a manifestation of the local Archimedean Langlands correspondence (see e.g. [1]). The analogy between mirror symmetry and local Archimedean Langlands correspondence looks not accidental and can eventually imply that local Archimedean Langlands correspondence follows from the mirror symmetry. 
In Section 4, the $q$-deformed $\Gamma$-function was represented as a partition of an equivariant three-dimensional topological linear sigma model on $D \times S^{1}$. The functional integral reduces to the functional integral over the fields on the boundary $T^{2}=S^{1} \times S^{1}$. On the other hand, the $q$-deformed $\Gamma$-function can be identified with a partition function of a chiral scalar field on $T^{2}$. This relation between topological theory on a three-dimensional manifold and holomorphic theory on its boundary resembles the relation between conformal blocks in Wess-Zumino-Witten (WZW) theory and Chern-Simons (CS) theory $[16,48]$. Such an analog of WZW/CS correspondence deserves considerations.

Let us note that the proposed functional integral representation for the classical $\Gamma$-function allows a straightforward quantization providing the $q$-deformed $\Gamma$-function. On the other hand, the standard construction of the $q$-deformed $\Gamma$-function in the classical setting is ad hoc. One can hope that further development of our approach would provide a canonical construction of $q$-deformations of other classical special functions.

Local $L$-factors and their $q$-counterparts are basic building blocks in the description of semi-infinite periods associated with a type $A$ topological sigma model with the target space $\mathbb{P}^{\ell}$ and more generally a homogeneous space of a classical Lie group $[22,23]$. The proposed functional integral representations should lead to a direct derivation of the results of [23] in the framework of topological sigma models in two- and three-dimensions.

Finally, let us stress that the main driving force of the whole project including this note and the previous ones [20-23] is to uncover the proper geometric description of Archimedean places in arithmetic geometry. The results of this note imply that the infinite-dimensional symplectic geometry could be a proper setting to discuss quantum field theory models for Archimedean arithmetic geometry seriously.

\section{Acknowledgments}

The research was supported by Grants RFBR-08-01-00931-a, 09-01-93108NCNIL-a. AG was also partly supported by Science Foundation Ireland grant. The research of SO was partly supported by P. Deligne's 2004 Balzan prize in Mathematics.

\section{Appendix A: Gaussian functional integral}

In the appendix, we describe the standard approach to the calculation/ definition of the Gaussian functional integrals using $\zeta$-function regularization 
$[32,38]$ (see also [44]). We start with a simple finite-dimensional Gaussian integrals

$$
I_{N}^{\mathbb{C}}(A)=\left(\frac{\imath}{2}\right)^{\ell+1} \int_{\mathbb{C}^{N}} \mathrm{e}^{-\frac{1}{2} \sum_{i, j=1}^{N} \bar{z}_{i} A_{i j} z_{j}} \prod_{j=1}^{N} d z^{j} d \bar{z}^{j}=\frac{1}{\operatorname{det} A / 2 \pi},
$$

where matrix $A$ is positively defined, i.e., is unitary equivalent to the diagonal matrix with positive eigenvalues. More generally, the Gaussian integral (A.1) for $A$ having complex eigenvalues $a_{j}$ such that $\operatorname{Re}\left(a_{j}\right) \geq 0, j=$ $1, \ldots, N$ is defined as a limit of the integral for $A$ having complex eigenvalues $a_{j}$ such that $\operatorname{Re}\left(a_{j}\right)>0, j=1, \ldots, N$. The resulting expression for $I_{N}^{\mathbb{C}}(A)$ coincides with the r.h.s. of (A.1). The following integral is an example of this more general case

(A.2) $I=\left(\frac{\imath}{2}\right)^{2} \int_{\mathbb{C}^{2}} d z d \bar{z} d w d \bar{w} \mathrm{e}^{-\lambda \frac{\imath}{2}(\bar{z} w+z \bar{w}+\bar{a} w+\bar{w} a)}=\left(\frac{2 \pi}{\lambda}\right)^{2}, \quad \lambda \in \mathbb{R}$.

Similar expression holds for Gaussian integral over anticommuting variables

$$
J_{N}^{\mathbb{C}}=\int_{\mathbb{C}^{\text {o|N }}} \mathrm{e}^{\imath \sum_{i, j=1}^{N} \bar{\eta}_{i} A_{i j} \eta_{j}} \prod_{j=1}^{N}\left(\imath d \eta^{j} d \bar{\eta}^{j}\right)=\operatorname{det} A
$$

where we use standard Berezin integration over anticommuting variables

$$
\int d \eta=0, \quad \int \eta d \eta=1
$$

and the sign convention in the multi-variable case is defined by

$$
\int \eta_{1} \eta_{2} \cdots \eta_{N} d \eta_{1} \cdots d \eta_{N}=1
$$

Let $\mathbb{C}^{N \mid N}$ be a linear super-space with the space of polynomial holomorphic functions $\mathbb{C}\left[z_{1}, \ldots, z_{N}, \eta_{1}, \ldots, \eta_{N}\right]$. We have a canonical measure on this space

$$
d m(z, \eta)=\left(d z_{1} d \bar{z}_{1} d z_{2} d \bar{z}_{2} \cdots d z_{N} d \bar{z}_{N}\right)\left(d \eta_{1} d \bar{\eta}_{1} d \eta_{2} d \bar{\eta}_{2} \cdots d \eta_{N} d \bar{\eta}_{N}\right)
$$

Then using the measure (A.3) the integral (A.1) can be rewritten as follows:

$$
I_{N}^{\mathbb{C}}(A)=\int_{\mathbb{C}^{N \mid N}} d m(z, \eta) \mathrm{e}^{-\frac{1}{2} \sum_{i, j=1}^{N} \bar{z}_{i} A_{i j} z_{j}-\frac{\imath}{2} \sum_{j=1}^{N} \bar{\eta}_{j} \eta_{j}}=\frac{1}{\operatorname{det} A / 2 \pi} .
$$


In particular, an analog of (A.2) is given by

$$
\begin{aligned}
\tilde{I} & =\frac{1}{(2 \pi)^{2}} \int_{\mathbb{C}^{2 \mid 2}} d z d \bar{z} d w d \bar{w} d \eta d \bar{\eta} d \xi d \bar{\xi} \mathrm{e}^{-\lambda \frac{\imath}{2}(\bar{z} w+z \bar{w}+\bar{a} w+\bar{w} a)+(\bar{\eta} \xi+\bar{\xi} \eta)} \\
& =1, \quad \lambda \in \mathbb{R}
\end{aligned}
$$

We need certain infinite-dimensional analogs of the integrals considered above. Instead of matrix $A$ we have some differential operator acting in an infinite-dimensional space of functions. To define corresponding infinitedimensional Gaussian integrals one should define a notion of the determinant of the corresponding differential operator. Let $\mathcal{D}$ be an operator with the positive discrete spectrum with finite multiplicities

$$
0<d_{0} \leq d_{1} \leq d_{2} \leq \cdots
$$

Consider $\zeta$-function for the operator $\mathcal{D}+\lambda$

$$
Z_{\mathcal{D}}(s, \lambda)=\sum_{n=0}^{\infty} \frac{1}{\left(d_{n}+\lambda\right)^{s}}
$$

where $s \in \mathbb{C}$ is such that the sum is convergent. The sum (A.6) can be continued to a meromorphic function of $s$. The regularized determinant of the operator $\mathcal{D}+\lambda$ is then defined as follows

$$
\ln \operatorname{det}(\mathcal{D}+\lambda)=-\left.\partial_{s} Z(s, \lambda)\right|_{s=0}
$$

Consider a special case of this construction for the operator $\mathcal{D}$ with the spectrum $d_{n}=\rho n, n \in \mathbb{Z}_{\geq 0}$. Corresponding $\zeta$-function

$$
\zeta_{\rho}(s, \lambda)=\sum_{n=0}^{\infty} \frac{1}{(\rho n+\lambda)^{s}}, \quad-\pi<\arg (\rho n+\lambda) \leq \pi,
$$

is basically the Hurwitz $\zeta$-function and has analytic continuation to all $s \in$ $\mathbb{C} /\{1\}$. We have

$$
\zeta_{\rho}(0, \lambda)=\frac{1}{2}-\frac{\lambda}{\rho}, \quad \partial_{s} \zeta_{\rho}(0, \lambda)=-\left(\frac{1}{2}-\frac{\lambda}{\rho}\right) \ln \rho+\ln \frac{1}{\sqrt{2 \pi}} \Gamma\left(\frac{\lambda}{\rho}\right) .
$$

Thus for the regularized determinant of $\mathcal{D}+\lambda$, we obtain

$$
\left[\prod_{n=0}^{\infty}(\rho n+\lambda)\right]_{\mathrm{reg}}=\rho^{1 / 2-\lambda / \rho} \frac{(2 \pi)^{1 / 2}}{\Gamma(\lambda / \rho)}
$$


We also define the regularized determinant of the operator given by multiplication by $\rho$ as

$$
\left[\prod_{n=0}^{+\infty} \rho\right]_{\mathrm{reg}}=\exp (\zeta(0,0) \ln \rho)=\rho^{\frac{1}{2}}
$$

Lemma A.1. The following identity holds

$$
\left[\prod_{n \in \mathbb{Z}}(\rho n+\lambda)\right]_{\mathrm{reg}}=1-e^{2 \pi \imath \lambda / \rho}, \quad \operatorname{Im}(\rho)>0 .
$$

Proof. We have

$$
\zeta_{\rho}^{*}(s, \lambda):=\sum_{n \in \mathbb{Z}} \frac{1}{(\rho n+\lambda)^{s}}=\zeta_{\rho}(s, \lambda)+\zeta_{-\rho}(s, \lambda)-\lambda^{-s}
$$

Simple calculation gives

$$
\begin{aligned}
\left.\frac{\partial}{\partial s} \zeta_{\rho}^{*}(s, \lambda)\right|_{s=0} & =\zeta_{\rho}^{\prime}(0, \lambda)+\zeta_{-\rho}^{\prime}(0, \lambda)+\ln \lambda \\
& =-\frac{\imath \pi}{2}-\imath \pi \frac{\lambda}{\rho}+\ln \left(\frac{1}{2 \pi} \Gamma\left(\frac{\lambda}{\rho}\right) \Gamma\left(1-\frac{\lambda}{\rho}\right)\right)
\end{aligned}
$$

Finally, we obtain

$$
\begin{aligned}
{\left[\prod_{n \in \mathbb{Z}}(\rho n+\lambda)\right]_{\mathrm{reg}} } & =\exp \left(-\left.\frac{\partial}{\partial s} \zeta_{a}^{*}(s, \lambda)\right|_{s=0}\right) \\
& =e^{\imath \pi \lambda / \rho} \frac{2 \pi \imath}{\Gamma\left(\frac{\lambda}{\rho}\right) \Gamma\left(1-\frac{\lambda}{\rho}\right)}=\left(1-e^{2 \pi \imath \lambda / \rho}\right)
\end{aligned}
$$

where we use the identity

$$
\Gamma(1-z) \Gamma(z)=\frac{\pi}{\sin (\pi z)}
$$

Remark A.1. Note that the regularization in Lemma A.1 is different from a more standard regularization which uses the Fredholm definition of the infinite determinants (see e.g. [44]). 


\section{References}

[1] J. Adams, D. Barbash and D. A. Vogan Jr., The Langlands classification and irreducible characters of real reductive groups, Progress in Mathematics, 104, Birkhäuser Boston, Inc., Boston, MA (1992).

[2] L. Alvarez-Gaume, Supersymmetry and the Atiyah-Singer index theorem, Commun. Math. Phys. 90(2) (1983), 161-173.

[3] O. Alvarez, I. M. Singer and P. Windey, Quantum mechanics and the geometry of the Weyl character formula, Nucl. Phys. B337(2) (1990), 467-486.

[4] P. S. Aspinwall and D. R. Morrison, Topological field theory and rational curves, Commun. Math. Phys. 151(2) (1993), 245-262.

[5] M. Atiyah and R. Bott, The momentum map and equivariant cohomology, Topology 23(1) (1984), 1-28.

[6] A. A. Beilinson, Height pairings between algebraic cycles, in 'K-theory, arithmetic and geometry', (ed. Yu. I., Manin), Moscow 1984/86, Lect. Notes Math., 1289, Springer, Berlin, 1987, 1-25, and Contemp. Math. 67 (1987), 1-24.

[7] M. Bershadsky, S. Ceccotti, H. Ooguri and C. Vafa, Kodaira-Spencer theory of gravity and exact results for quantum string amplitudes, Commun. Math. Phys. 165 (1994), 311-427, [hep-th/9601014].

[8] J.-M. Bismut, The Atiyah-Singer theorems: a probabilistic approach, II, J. Funct. Anal. 57 (1984), 329-348.

[9] D. Bump Automorphic forms and representations, Cambridge University Press, Cambridge, 1998.

[10] W. Casselman and J. Shalika, The unramified principal series of padic groups II. The Whittaker function. Compositio Math. 41 (1980), 207-231.

[11] S. Cordes, G. Moore and S. Ramgoolam, Lectures on 2D YangMills theory, equivariant cohomology and topological field theories, in 'Proceedings of the 1994 Les Houches school on fluctuating geometries', Nucl. Phys. Proc. Suppl. 41 (1995), 184-244, [arXiv: hep-th/9411210].

[12] P. Deligne, Theorie de Hodge I, in 'Actes du Congrès International des Mathematiciens', Nice, 1970, 1, Gauthier-Villars, Paris, 1971, 425-430. 
[13] C. Deninger, On the $\Gamma$-factors attached to motives, Invent. Math. 104 (1991), 245-261.

[14] C. Deninger, Local L-factors of motives and regularized determinants, Invent. Math. 107 (1992), 135-150.

[15] J. J. Duistermaat and G. J. Heckman, On the variation in the cohomology of the symplectic form of the reduced phase space, Invent. Math. 69(2) (1982), 259-268.

[16] S. Elitzur, G. Moore, A. Schwimmer and N. Seiberg, Remarks on the canonical quantization of the Chern-Simons-Witten theory, Nucl. Phys. B326 (1989), 108.

[17] D. Friedan and P. Windey, Supersymmetric derivation of the AtiyahSinger index theorem and the chiral anomaly. Nucl. Phys. B235 (1984), 395-416.

[18] J.-M. Fontaine, Le corps des périodes p-adiques, Astérisque 223 (1994), 59-111.

[19] A. Gerasimov, Quantization of special geometry and holomorphic anomaly equation, Talk at Frobenius manifolds, quantum cohomology, and singularities, MPIM, Bonn, July 2002, Towards integrability of topological strings, Arbeitstagung 2005 talk, MPIM, Bonn, Preprint: MPIM2005-60q, available at http: //www.mpim-bonn.mpg.de/preprints/.

[20] A. Gerasimov, D. Lebedev and S. Oblezin, New integral representations of Whittaker functions for classical groups, [arXiv:math.RT/0705.2886].

[21] A. Gerasimov, D. Lebedev and S. Oblezin, Baxter operator and Archimedean Hecke algebra, Commun. Math. Phys. 284(3) (2008), 867-896, [arXiv:math.RT/0706.347].

[22] A. Gerasimov, D. Lebedev and S. Oblezin, Baxter Q-operators and their Arithmetic implications, Lett. Math. Phys. 88(1-3) (2009), 3-30, [arXiv:math.RT/0711.2812].

[23] A. Gerasimov, D. Lebedev and S. Oblezin, On q-deformed $\mathfrak{g l}_{\ell+1^{-}}$ Whittaker functions I,II,III, Commun. Math. Phys. 294 (2010), 97-119, [arXiv:0803.0145]; Commun. Math. Phys. 294 (2010), 121-143, [arXiv:0803.0970]; Lett. Math. Phys. 97 (2011), 1-24, [arXiv:0805.3754]. 
[24] A. Gerasimov, D. Lebedev and S. Oblezin, Archimedean L-factors and topological field theories II, Commun. in Number Theory and Physics, 5 (1) 2011, [arXiv:0906.1065].

[25] A. Gerasimov, S. Kharchev, D. Lebedev and S. Oblezin, On a Gauss-Givental representation for quantum Toda chain wave function, Int. Math. Res. Notices (2006) Article ID 96489, 23 p. [arXiv:math.RT/0505310].

[26] A. Gerasimov and S. Shatashvili, On string field theory description of non-critical $c<1$ strings, to appear.

[27] A. Givental, Homological geometry I. Projective hypersurfaces, Selecta Math., New Series Volume 1, 2, (1995), 325-345.

[28] A. Givental, Equivariant Gromov-Witten invariants, Internat. Math. Res. Notices no. 13 (1996), 613-663, [arXiv:alg-geom/9603021].

[29] A. Givental, On the WDVV-equation in quantum K-theory, Michigan Math. J. 48 (2000), 295-304, [arXiv:math/0003158].

[30] A. Givental, Stationary phase integrals, quantum toda lattices, flag manifolds and the mirror conjecture, in 'Topics in singularity theory', Amer. Math. Soc. Transl. Ser. 2, 180, American Mathematical Society, Providence, Rhode Island, 1997, 103-115, [arXiv:alg-geom/9612001].

[31] A. Givental and Y.-P. Lee, Quantum K-theory on flag manifolds, finite-difference Toda lattices and quantum groups, Invent. Math. 151 (2003), 193-219, [arXiv:math.AG/0108105].

[32] S. W. Hawking, Zeta function regularization of path integrals in curved space time, Commun. Math. Phys. 55 (1977), 133-148.

[33] K. Hori and C. Vafa, Mirror symmetry, [arXiv:hep-th/0002222].

[34] M. Kontsevich, Intersection theory on the moduli space of curves and the matrix Airy function, Commun. Math. Phys. 147 (1992), 1.

[35] M. Kontsevich and Yu. Manin, Gromov-Witten classes, quantum cohomology and enumerative geometry, Commun. Math. Phys. 164 (1994), 525-562, [arXiv:hep-th/9402147].

[36] J. Bernstein and S. Gelbart (eds.), An introduction to the Langlands program, Lectures presented at the Hebrew University of Jerusalem, 
Jerusalem, 12-16 March, 2001, Birkhäuser Boston, Inc., Boston, MA, 2003.

[37] Yu. Manin, Lectures on zeta functions and motives (according to Deninger and Kurokawa), in Columbia University Number Theory Seminar, Astérisque, 228 (1995), 121-164.

[38] D. B. Ray and I. M. Singer, Analytic torsion for complex manifolds, Ann. Math. 98 (1973), 154-177.

[39] P. Deligne, P. Etingof, D. Freed, L. Jeffrey, D. Kazhdan, J. Morgan, D. Morrison, E. Witten (eds.), Quantum fields and strings: a course for mathematicians, American Mathematical Society, Providence, RI, 1999.

[40] T. Shintani, On an explicit formula for class 1 Whittaker functions on $G L_{n}$ over p-adic fields. Proc. Japan Acad. 52 (1976), 180-182

[41] J. P. Serre, Facteurs locaux des fonctions zêta des variétés algébraiques (définisions et conjecures). Sém. Delange-Pisot-Poitou, exp. 19, $(1969 / 70)$.

[42] J. Tate, Number theoretic background, in Automorphic forms and L-functions, Proc. Symp. Pure Math. 33 (1979), part 2, 3-26.

[43] P. Vojta, Diophantine approximations and value distribution theory, Lect. Notes Math. 1239, Springer-Verlag, Berlin, 1987.

[44] A. Voros, Spectral functions, special functions and the selberg zeta function, Commun. Math. Phys. 110 (1987), 439-465.

[45] E. Witten, Topological Sigma Model, Commun. Math. Phys. 118(3) (1988), 411-449.

[46] E. Witten, Mirror manifolds and topological field theory, in 'Essays on mirror manifolds', Internat. Press, Hong Kong, 1992, 120-158, [arXiv:hep-th/9112056].

[47] E. Witten, Two dimensional Gauge theories revisited, J. Geom. Phys. G9 (1992), 303-368, [arXiv:hep-th/9204083].

[48] E. Witten, Quantum field theory and the Jones polynomial, Commun. Math. Phys. 121 (1988), 351-399. 
Institute for Theoretical and Experimental Physics 117259, Moscow

Russia

School of Mathematics

Trinity College

DUBLIN 2

IRELAND

Hamilton Mathematics Institute

Trinity College

DUBLIN 2

IRELAND

Received July 29, 2010 